\title{
V. Im „Zick-Zack“ zum Sozialismus? Die DBD und die agrarpolitische und agrarsoziale Entwicklung von Ende 1948 bis 1952
}

Eine Analyse ausgewählter agrarpolitischer und -sozialer Vorgänge von 1948 bis 1952 unter dem Blickwinkel der Rolle der DBD lohnt aus verschiedenen Gründen. Denn im Einzugsbereich der DBD sammelten sich agrarische und soziale Problemkonstellationen der ländlichen Gesellschaft wie in einem Brennglas. Bis 1950 integrierte ihre Mitgliederbasis im Prinzip alle Gruppen bäuerlicher Bevölkerung und darüber hinaus auch weitere Teile der Dorfbewohner jenseits der klassenkämpferisch vorgegebenen Trennlinien unter der Bauernschaft ${ }^{1}$. Obgleich sich die Mitglieder der DBD wie die der SED durch eine "fortschrittliche" Gesinnung auszeichnen sollten, wurden den DBD-Kollegen im Unterschied zu den bäuerlichen SED-Genossen bewußt Freiräume zugebilligt. So erleichterte es die Verankerung im Dorf, daß bei der Parteiaufnahme keine Angaben zur Konfession erhoben wurden; auch eine Mitgliedschaft in der NSDAP wurde hierzu in den Jahren 1948/49 nicht systematisch erfragt. Einer sorgfältigen Überprüfung wurden in der Regel erst jene unterzogen, die parteïntern durch die Übernahme von Funktionen aufstiegen oder eine Parteischule besuchten. Obwohl der Anleitungsapparat der SED bis 1952 erhebliche Lücken aufwies, befand sich die DBD in einer prinzipiellen Abhängigkeit. Außerdem hatte sie seit der Staatsgründung Regierungsverantwortung übernommen. Eine ganze Reihe von Maßnahmen der staatlichen Exekutive wurde im DBD-geleiteten Ministerium für Land- und Forst- und Nahrungsgüterwirtschaft (MLF) in Abstimmung mit der SED-Landwirtschaftsabteilung ausgearbeitet und war von der Regierung durchzuführen, selbst wenn die DBD an der eigentlichen Konzeption unbeteiligt war. Die eingeschlagene Agrarpolitik mußte sie jedoch auch gegenüber ihren eigenen Mitgliedern verantworten.

\section{Sowjetische Kursbestimmung im Dezember 1948}

Während der zweiwöchigen Reise der SED-Führungsspitze im Dezember 1948 nach Moskau, die der Koordinierung der für Anfang 1949 angesetzten 1. Parteikonferenz der SED diente, erfolgten ganz grundsätzliche Festlegungen zum agrarpolitischen Kurs. Im Grunde lesen sich Piecks Aufzeichnungen wie eine Handlungsanleitung für das agrarpolitische Vorgehen bis 1951. Pieck notierte nach der Besprechung, aber offenbar noch in Moskau und damit in zeitlicher

1 Siehe dazu Tabellen 7 und $10 \mathrm{im}$ Anhang. 
Nähe zu den von Stalin angeordneten Kurskorrekturen folgendes zur landwirtschaftlichen Lage:

„Gesetz nicht bearb. Boden wird eingezogen Gesetz über Landarbeiter Lage

Widersprüche zwischen Arb.tag Landarb. u. Großbauern

Gesetz Masch.Stationen staatlich von $\mathrm{VdgB}$ abkaufen

Keine Enteignung, noch zu früh in SU 1925/27

Vorstufe zu Kollektivisierung noch keine Volksdemokratie nicht gegen Gruppen von Besitzern vorgehen nur gegen einzelne, wenn diese Sabotage durch ökonomische Mittel regulieren [...] Vertrag statt Kontrolle nicht direkte Eingriffe, sondern Zickzack [...]

Lage nicht gleich wie VD [Volksdemokratien] noch kein einheitl. Staat stehen nicht vor der Macht Einheit Frieden Preissenkung Erhöhung Löhne Ernährung verbessern [...] Nicht auf $\underline{\text { Plan }}$ pochen, differenzieren [...] Ablieferungssystem elastisch Kleinbauern begünstigen Maschinen-Stationen verstaatlichen zu pol. Zentren machen - wie SU 1926/27 Landwirtsch. Genossensch. säubern"2.

Stalin schärfte der SED-Führung ein, daß sich die SBZ noch nicht im Stadium einer Volksdemokratie befinde ${ }^{3}$. Daher lehnte er eine offene Enteignungspolitik insgesamt, also auch in der Landwirtschaft, ab. Diese Kernaussage übermittelte Pieck zwar dem Zentralsekretariat, allerdings blieb er seinen Aufzeichnungen zufolge bezüglich der Landwirtschaft in einigen Punkten unpräzise. So unterschlug er Stalins Vergleich mit der Entwicklung in der Sowjetunion, demzufolge sich die SBZ in einem Stadium deutlich vor der Kollektivierung befinde, und er führte statt Maschinen-Ausleih-Stationen (MAS) den Begriff Maschinen-Traktoren-Stationen (MTS) ein - so hieß die sowjetische Variante-die als politische Zentren auszubauen seien ${ }^{4}$. Im Hinblick auf die Gestaltung der landwirtschaftlichen Eigentumsformen häuften sich in der SED - besonders in der SED-gesteuerten VdgB - ab Oktober 1948 Indizien für ein aktives Vorantreiben jener agrarpolitischen Elemente, die als Keimzellen des Agrarsozialismus angesehen wurden. So hielten offenbar vor allem die VdgB-Funktionäre den "Klassenkampf“ auf dem Land nicht nur verbal für ausgebrochen ${ }^{5}$, sondern sie traten ihrerseits für die Bildung von „Produktivgenossenschaften" ein und hatten sogar schon Vorbereitungen dafür getroffen. Da es auch Hinweise für sowjetische Überlegungen zu einer landwirtschaftlichen Sozialisierung 1948 in der SBZ gibt, kann man davon ausgehen, daß die verantwortlichen SED-Funktionäre bis zu Stalins Schelte nicht im Alleingang handelten, sondern durchaus mit Rückendeckung zumindest von Teilen der SMAD6.

2 Badstübner/Loth (Hg.), Wilhelm Pieck-Aufzeichnungen, Besprechung am 18. 12. 1948, S. 259-263, Zitat S. 260f., eckige Klammern von Verfasserin, andere Hervorhebungen lt. Edition.

3 Loth, Stalins ungeliebtes Kind, S. 145-148; Staritz, SED, Stalin und der „Aufbau des Sozialismus", S. $691 \mathrm{f}$.

4 Badstübner/Loth (Hg.), Wilhelm Pieck-Aufzeichnungen, Notizen Piecks vom 27. 12. 1948 für den Bericht über die Besprechung vom 18. 12. 1948 vor dem ZS der SED, S. 265274, hier S. 268.

5 So auch Naimark, Russen, S. 209.

6 Ebd., S. 207 f. Ein Schreiben Korbuts, 1948 Chef der Abt. für Land- und Forstwirtschaft 
Besonders Kurt Vieweg, seit August 1947 Generalsekretär der VdgB, machte sich für die Übernahme agrarpolitischer Modelle stark, wie sie in der benachbarten Volksdemokratie Polen seit Sommer 1948 praktiziert wurden ${ }^{7}$. Als Ergebnis eines Besuchs in Polen brachte er ein Programm mit, das neben der Einrichtung von "Produktivgenossenschaften" auch ein ausgeklügeltes Vorgehen gegen sogenannte "Großbauern" umfaßte, also gegen jene Gruppe, die nach marxistischer Ideologie den "Klassenkampf" gegen den Sozialismus anfachte und daher zu bekämpfen war ${ }^{8}$. Im Landkreis Dresden waren die Planungen für die Errichtung von vier solchen "Produktivgenossenschaften" auf 15000 ha ehemaligem Stiftsgutsbesitz - offensichtlich in Absprache mit Vieweg - bereits fortgeschritten. Trotz gewisser Anklänge an eine gemeinwirtschaftliche Nutzung, wie sie im Zusammenhang mit der Bodenreform 1945/46 betrieben wurde, zielten diese Vorhaben auf eine Aufteilung der Ländereien in je Sieben-Hektar-Parzellen an Landarbeiter und "politisch ausgesuchte Umsiedler", die das Land dann jedoch gemeinschaftlich bewirtschaften sollten ${ }^{10}$. Vieweg drängte im SED-Agrarapparat auf eine Umsetzung der in Polen inspizierten Vorgehensweisen in der Agrarpolitik $^{11}$. Die im Zuge der Transformation der SED 1948 mit besonderem Einfluß versehenen Betriebsgruppen, hier jene der HVLF, dienten auch der Disziplinierung von Abweichlern. Der altgediente SED-Agrarfunktionär Edwin Hoernle, der gegen diese Radikalisierung offenbar Vorbehalte hegte, wurde vor der SEDBetriebsgruppe der HVLF im Oktober zur Rechenschaft gezogen, verlor daraufhin an Einfluß und gab die Leitung der Hauptverwaltung $1949 \mathrm{ab}^{12}$.

Das legt den Schluß nahe, daß führende Vertreter der VdgB, namentlich Vieweg, und die SED-Spitze beabsichtigten, die schon seit dem Frühjahr 1948 intern diskutierten Attacken gegen die "Großbauern"13 im Herbst 1948 in eine Politik

der SMAD, an Semjonow vom 17. 9. 1948 forderte, daß man die Kominform-Resolution zu Jugoslawien und die Resolution der polnischen Arbeiterpartei PPR als Inspiration prüfen solle. Hingegen wurden in die DBD eingetretene SED-Politiker im Juli 1948 für diese Bezugnahme kritisiert; Bonwetsch u.a. (Hg.), Politik, S. $167 \mathrm{f}$.

7 Zur Kollektivierung in Polen seit Sommer 1948: Jarosz, Polityka, S. 54-89, Fejtö, Geschichte, Bd. 1, S. 360-382; Mrowka, Bodenreform, S. 240 ff. Zu SMAD-Kontakten Viewegs im Oktober 1948: Scholz, Bauernopfer, S. 76.

8 BA DC-15/737, Bl. 399-415, Bericht über eine Reise Viewegs nach Polen, am 6. 12.1948 an Rau, Bl. $414 \mathrm{f}$. Vorschläge, die in der SBZ "trotz der anders gearteten Bedingungen“" sofort angewendet werden könnten, waren u.a. eine Revision des ldw. Steuersystems. Der Bericht betonte dazu, daß die polnische Partei als „Hauptmittel zu[r] Durchführung des Klassenkampfes auf dem Lande“ die Einführung einer stark progressiven Steuerpolitik sehe (Bl. 402). Ohne diese Vorreiterrolle Viewegs: Scholz, Bauernopfer, S. 74-78.

9 Vgl. Kuntsche, "Gemeinwirtschaft“; im Dez. 1945 setzte eine Kampagne gegen die Gemeinwirtschaft ein, die im Feb. 1946 ihren Höhepunkt erreichte (ebd., Bd. 2, S. 212 f.).

10 SAPMO VdgB 136, Schreiben, vermutlich von Genossen Schenk, HA-Leiter II (der VdgB?), 25. 11. 1948.

11 BA DC-15/210, Bl. 2-4, Protokoll über Besprechung u.a. mit Hoernle u. Reutter in der HVLF, 12. 11. 1948. Vgl. zu Stiftsgütern Zinke, Transformation, S. 174-181.

12 Bauerkämper, Wege, S. 259.

13 Reutter lehnte im Januar 1948 einen Angriff auf alle Altbauern, wie ihn Ernst Busse, $\mathrm{VdgB}$, zu dieser Zeit vertreten hatte, noch ab, Bauerkämper, Wege, S. 259. Die Existenz der Debatte belegt dies nur. 
aktiver Bekämpfung bei gleichzeitiger Förderung der Kleinbauern zu überführen. Das Vorgehen gegen die "Großbauern“" war nun begleitet von konkreten Experimenten zur Einführung von Modellen der kollektiven Bewirtschaftung; eine Überführung in eine kollektive Eigentumsstruktur war für diese Form von „Produktivgenossenschaften " allerdings noch nicht vorgesehen ${ }^{14}$. Bekanntlich hatte die SED bis 1948 einen moderaten Kurs gegenüber den "Großbauern" vertreten, der diese weder ausdrücklich noch systematisch bekämpfte, solange sie ihre Ablieferungspflicht erfüllten und sich politisch nicht gegen die SED engagierten ${ }^{15}$. Die in der älteren Forschung überwiegend vertretene Meinung, im Herbst 1948 habe es eine Zäsur in der Bündnispolitik gegenüber den "Großbauern" gegeben, vereinfacht die Situation und setzt den Einschnitt zu spät an. Der agrar- und bündnispolitische Handlungskontext hatte sich zwischen Frühjahr und Herbst 1948 durch die Gründung einer Bauernpartei für die SED verändert. Da jetzt eine Partei neben der SED existierte, die prinzipiell alle bäuerlichen Schichten einbinden sollte, konnte die SED ihr eigenes Vorgehen radikalisieren. Der DBD fiel der Part zu, sowohl die Radikalisierung mitzutragen, vor allem aber sollte sie diese dadurch flankieren, daß sie sich in ihrem agrarpolitischen Handeln weiterhin allen bäuerlichen Gruppen zuwandte.

Auch die DBD wurde im Herbst 1948 in Richtung gegen die "Großbauern" in einer Art volksdemokratischem Attentismus angeleitet. Bis dahin hatte sie sich programmatisch nicht öffentlich gegen die "Großbauern" ausgesprochen, vielmehr in ihren ersten Programmaussagen die gesamte "bäuerliche Bevölkerung“ als potentielle Mitglieder umworben. Noch Ende Oktober 1948 war das Sekretariat der DBD unsicher darüber, wie sich die Partei in der "Großbauernfrage" verhalten sollte ${ }^{16}$. Die Parteivorstandssitzung vom 3. Dezember 1948 klärte diese Frage dann zumindest auf oberer Ebene. Insgesamt spiegelt diese Zusammenkunft deutlich wider, wie sehr die DBD bemüht war, ihre politisch-ideologischen Debatten den Anforderungen von SED und SMAD anzupassen. Man nahm Goldenbaums Bericht über eine Reise in die Sowjetunion zum Anlaß, um ideologische Kernthemen abzuhandeln und so die richtige politische Gesinnung unter Beweis zu stellen ${ }^{17}$. Die SMAD erwartete noch im gleichen Monat einen Vorschlag der DBD zum Aufbau eines Parteischulungswesens.

14 Siehe dazu auch Beschlußentwurf der Betriebsgruppe der HVLF in der DWK, undatiert [lt. Zusammenhang unmittelbar nach 13.11. 1948], SAPMO VdgB 136; er sah u.a. den "sofortige[n] Ausschluss aller Grossbauern aus der Partei“ vor und die „Ausschaltung des privaten Grosshandels bei Erfassung, Lagerung, Vermittlung und Verkauf landwirtschaftlicher Erzeugnisse“.

15 Gegen Piskol, Entwicklung, S. 419, ist jedoch einzuwenden, daß die Großbauern schon vor dem Herbst 1948 nicht mehr als "Verbündete“ behandelt worden waren. In der Argumentation zur Großbauernpolitik widersprüchlich Piskol/Nehrig/Trixa, Umwälzung, S. $153 \mathrm{f} .$, S. $161 \mathrm{f}$.

16 ACDP VI-052-0519, Protokoll Sekretariat, 26. 10. 1948.

17 ACDP VI-052-0141, Protokoll PV, 3. 12. 1948. Albrecht sprach zur Aktivistenbewegung und zog Parallelen zur Stachanow-Bewegung; Helmschrott klärte den Begriff des Kollektiv-Eigentums; Griebel erläuterte das „neue Verhältnis der Arbeiterklasse zu den Bauern“. 
Es ist in der Forschung strittig, inwiefern dieser volksdemokratische Kurs von der SED-Führungsspitze unter Ulbricht gemeinsam mit Teilen der SMAD um Tjulpanow ohne Rückkoppelung mit Stalin oder gar gegen dessen politische Absicht verfochten wurde ${ }^{18}$. Prinzipiell hatte Stalin nichts gegen eine Transformation der Agrarverfassung, die sich an sozialistischen Zielen ausrichtete, einzuwenden. Im Gegenteil, Keimzellen sozialistischer Agrarpolitik sollten gefördert werden, damit die SBZ auf Dauer nicht mehr aus ernährungspolitischen und volkswirtschaftlichen Gründen auf die Produktion durch „kapitalistische Elemente" angewiesen war. In der Agrarpolitik der SBZ und dann der DDR liegt hier kein Bruch vor, sondern diese Linie wurde kontinuierlich verfolgt. Die Ernährungswirtschaft sollte nach einer technischen und finanziellen sowjetischen Starthilfe selbstläufig funktionieren und langfristig auf Kredite verzichten können. Den „kapitalistischen“ Teilen, und dazu zählte auch Stalin die „Großbauern", sollten daher erhebliche Lasten auferlegt werden, die der agrarmarxistischen Ideologie zufolge deren politische $\mathrm{Kraft}$ zugleich mit der wirtschaftlichen auslöschten. Diese Konzeption kollidierte keineswegs mit einer gesamtdeutschen Perspektive. Im Gegenteil, auch das Standbein SBZ sollte in seinen ordnungspolitischen Rahmenbedingungen für die Agrarwirtschaft derart sozioökonomisch transformiert werden, daß es im Falle eines deutschen Einheitsstaates eine Modellfunktion übernehmen konnte. Dies erforderte die Errichtung eines volkswirtschaftlich tragfähigen Gegenpols zur herkömmlichen, unter "großbäuerlichem“ Einfluß stehenden Landwirtschaft, mit dessen Hilfe die langfristigen Strukturprobleme der Bodenreform politisch und ökonomisch aufgefangen werden sollten. Die Protagonisten dieses Gegenentwurfs sollten die klein- und mittelbäuerlichen Betriebe sowie die VEG sein. Mit der VdgB, die zumindest in der Führung von sozialistischem Gedankengut durchdrungenen war, stellte man ihnen eine genossenschaftliche Partnerin an die Seite. Die Maschinen-Ausleihstationen sollten ihnen den erforderlichen betriebstechnischen Rückhalt liefern. Beide hatten auch einen politischen Erziehungsauftrag.

In der historischen Situation des Dezember 1948 korrigierte Stalin jedoch entscheidend die Art des geplanten Vorgehens der SED. Denn die Maßnahmen in der SBZ mußten zu diesem Zeitpunkt unbedingt mit seiner auf Gesamtdeutschland bezogenen Politik vereinbar sein. Die Einschätzung von Teilen der SED-Agrarfunktionäre, unmittelbar vor einer „Kollektivisierung“ zu stehen, rückte Stalin daher ganz klar zurecht, indem er das agrarpolitische Stadium der SBZ mit den Zuständen in der Sowjetunion Mitte der zwanziger Jahre verglich, also vor der offensiven Kompaktkollektivierung, die bekanntlich ab 1929 einsetzte ${ }^{19}$. Wenn wir Piecks Niederschrift Glauben schenken können, so schloß Stalin kollektive Wirtschaftsformen für Ostdeutschland nicht prinzipiell aus. Die Notizen geben grundsätzlich eine agrarpolitische Richtung Stalins wieder, die von Beginn an die Durchsetzung sozialistischer Verhältnisse begünstigen und dazu die kapitalistischen Strukturen eliminieren wollte. Allerdings war diese Vorgabe flexibel, beson-

${ }_{18} \mathrm{Zu}$ dieser Auffassung prononciert: Loth, Stalins ungeliebtes Kind, S. 129-148.

19 Grundlegend dazu Merl, Anfänge, und ders., Agrarmarkt. 
ders deutschlandpolitische oder volkswirtschaftliche Gründe konnten sie zeitweilig konterkarieren ${ }^{20}$.

Während die SED mit einer offenen, plumpen Enteignungspolitik liebäugelte, empfahl Stalin ein Instrumentarium an unterschiedlich scharfen Waffen gegen kapitalistische Einflüsse in der Agrarwirtschaft, wie ein „elastisches Ablieferungssystem" und die „Differenzierung“ der Planvorgaben nach Betriebsgrößengruppen. Beides sollte die Kleinbauernschaft begünstigen und andere bäuerliche Gruppen benachteiligen, insgesamt jedoch den ernährungswirtschaftlichen Bedarf sicherstellen. In der sozialistischen Utopie gefangen, vertraute man auf die Dynamik der Entwicklung und hoffte, die Förderung sozialistischer „Kernbereiche“ würde mit einem gewissen revolutionären Automatismus „ansteckend wirken“, die "Klassengegner" aber letztlich besiegen.

Die agrarpolitischen Mittel sind keineswegs alle primär vom klassenkämpferischen Impetus durchdrungen gewesen, wie es das zeitgenössische westdeutsche Schrifttum überwiegend behauptete, aber auch in der ostdeutschen Forschung zur opportunen Interpretation der Parteilinie gehörte ${ }^{21}$. Ihrer vorwiegenden Zweckbestimmung zufolge lassen sich die hier in einer Auswahl genannten Maßnahmen in drei Gruppen untergliedern: (1) eine Ad-hoc-Maßnahmenpolitik unter dem Primat der Ernährungssicherung, worunter auch die Wirtschaftsplanung fiel, (2) der Ausbau des staatlichen Sektors und (3) Bündnispolitik und „Klassenkampf“.

\section{Ernährungssicherung und Wirtschaftsplanung}

Eine Reihe von Regelungen zielte zunächst auf eine Produktionssteigerung zur Sicherung der Ernährung, taugte aber ebenso als Mittel im Klassenkampf mit vermeintlichen „Großbauern“. Die Vorgaben hatte der Mitte 1948 verabschiedete Zweijahrplan formuliert, der vorsah, bis 1950 die Versorgung mit Nahrungsmitteln deutlich zu erhöhen. Bis dahin sollten die Versorgungsnormen im Vergleich zu 1948 um knapp ein Drittel auf 2000 Kalorien pro Person und Tag gesteigert werden. Das Schwergewicht der Ressourcenverteilung im Zweijahrplan lag auf der Schwerindustrie und dem volkseigenen Sektor. Die daraus resultierende Vernachlässigung der Agrarwirtschaft, aber auch der Textilindustrie und des Wohnungsbaus bedeutete eine höhere Belastung für die gesamte Landwirtschaft, einschließlich der kleinen und mittleren Betriebe wie der Neubauern darunter, zugunsten des industriellen Wiederaufbaus ${ }^{22}$. In dieser Gewichtung hatten die ernährungswirtschaftlichen Belange Priorität. Stalins Hinweisen vom Dezember

20 Sogar Loth bezeichnet Stalins Widerspruch im Dezember 1948 als streng in der Sache, „inhaltlich aber etwas diffus". Loth, Stalins ungeliebtes Kind, S. 147.

21 Merkel/Schuhans, Agrarwirtschaft, S. 27-31, 36-86; Tümmler, Agrarpolitik, S. 12-20; Kramer, Bolschewisierung; Piskol/Nehrig/Trixa, Umwälzung; vgl. Bauerkämper, Legitimation, S. 40-49; ders., Amerikanisierung, bes. S. 196 f.; Kluge, „sozialistische Landwirtschaft", S. 11-38.

22 Vgl. dazu Zank, Wirtschaft, S. 66-80, 101-104. Piskol/Nehrig/Trixa, Umwälzung, S. $154 \mathrm{f}$., betonen die Zielrichtung des Zweijahrplanes hinsichtlich des Ausbaus des volkseigenen Sektors und einer als Ernährungspolitik verstandenen Agrarpolitik. 
1948 zufolge hatte dabei eine politische Ausrichtung, die die Lebensmittelzuteilung der Arbeiterklasse verbesserte und die Stimmung der Bevölkerung vor Wahlen stimulieren sollte, Vorrang.

$\mathrm{Zu}$ den Maßnahmen zählte die Einführung der Hektarveranlagung für die Ablieferung tierischer Produkte (Fleisch, Milch, Eier) zum 1. Januar 1949 anstelle der bisherigen Stückveranlagung ${ }^{23}$. Sie sollte insgesamt dem Aufbau eines Viehbestandes und damit einer besseren Eiweißversorgung dienen ${ }^{24}$. Dieser Wechsel belastete vor allem die größeren Betriebe zusätzlich, weil sie aus betriebswirtschaftlichen Gründen in der Regel mit steigender Fläche einen geringeren Viehbesatz pro Flächeneinheit aufwiesen - und nun gehalten waren, diesen zu erhöhen, um ihre Ablieferungsnorm zu erfüllen. Diese Betriebe waren häufig auf arbeitsintensive Hackfruchtkulturen, Saatzucht oder auch die Herdbuchhaltung spezialisiert, also Bewirtschaftungsformen, deren Erfolg maßgeblich von der Erfüllung von Qualitätsstandards abhängig war und daher weniger auf Quantität zielte. Die pauschale Hektarveranlagung und die damit einhergehende Erhöhung des Pflichtablieferungssolls schadete diesen Betrieben, weil sie deren betriebswirtschaftlicher Logik zuwiderlief25. In der "volksdemokratischen" Euphorie, die im Herbst 1948 in der SED-Landwirtschaftsabteilung herrschte, wurde die Hektarveranlagung daher als „äußerst wichtiges Mittel des Klassenkampfes gegen die Großbauern“ betrachtet ${ }^{26}$.

Obwohl die kleinen und mittleren Betriebe im Prinzip begünstigt wurden dies stellte die DBD im Hinblick auf ihre Klientel befriedigt fest -, litten auch in diesen Gruppen so manche Wirtschaften und Neubauernbetriebe unter dieser Veranlagungsform. Denn nur ein geringer Teil jener Betriebe hatte sich bis dahin wirtschaftlich konsolidiert. Die Mehrheit der Neubauernbetriebe war immer noch durch ungenügende Viehausstattung, mangelhafte Versorgung mit Maschinen und Geräten sowie eine unzureichende Ausstattung mit Gebäuden geschwächt, sollte aber unabhängig davon ihr Soll nach Hektaranzahl bestreiten ${ }^{27}$. Die Zahl der zurückgegebenen Neubauernbetriebe stieg weiter an - dies wurde

23 ZVOBl. 1949, T. 1, Nr. 11, S. 89.

24 Zur Diskussion in der SED und der Abstimmung mit Kowal (SMAD): SAPMO DY 30 IV 2/7/170, Bl. 94-96, Aussprache (Merker, Hoernle, Reutter, F. Scholz, Schellenberger) über Hektarveranlagung und Genossenschaftsfragen, 10.11. 1948. BA DC-15/715, Bl. 70, Antwort Kowal an Rau, 17. 11. 1948, er billigt im Prinzip den Übergang zur Hektarveranlagung. Nach Ansicht der SMAD müsse diese auf die Pflichtablieferung von Milch und Eiern ausgedehnt werden. Schulz, Probleme, S. 7.

${ }_{25} \mathrm{Merkel} / \mathrm{Schuhans,} \mathrm{Agrarwirtschaft,} \mathrm{S.} \mathrm{46;} \mathrm{siehe} \mathrm{auch} \mathrm{Piskol/Nehrig/Trixa,} \mathrm{Umwälzung,}$ S. $159 \mathrm{f}$.

26 Nehrig, Weiterentwicklung der agrarpolitischen Konzeption, S. 494.

27 Nehrig, Bauern; Bauerkämper, Von der Bodenreform, S. 123-125; speziell zu Mecklenburg: Bauerkämper, Antinomien, S. 369-376; die Fehlkonzeption und ökonomischen Folgen der Bodenreform teilweise verkennend: Nehrig, Entwicklung, S. 68-70, so wurden von rund 210000 Neubauernwirtschaften im Jahr 1949 rund 30000 aufgegeben, die meist wiederbesetzt werden konnten; weitere 30000 Neubauern gaben 1950/51 ihre Wirtschaften auf. Beim Auslaufen des Programms 1950 verfügten erst die Hälfte der Neubauernbetriebe über Wohnhaus, Stall und Scheune. Aktengestützte Zahlen auch bei Bell, Enteignungen, S. 16: Danach gaben 1946/47 14552, 1948 10531, 194911 103, 1950 16609, 1951 14250 und 195213587 Neubauern ihre Betriebe zurück. 
1951 mit juristischen Mitteln erschwert ${ }^{28}$ - oder die Neubauern verließen das Bodenreformland einfach ohne Absprachen. Auch mittlere Betriebe, die das gestiegene Soll in tierischen Produkten nicht erfüllen konnten, sich daher ersatzweise zur Ablieferung anderer Produkte verpflichteten, erlitten erhebliche finanzielle Einbußen. Infolge der höheren Pflichtabgaben verringerten sich ihre Einnahmen aus dem Verkauf von Produkten zum lukrativeren Preis der sogenannten „freien Spitzen “. Erst diese Erlöse gestatteten in der Regel betriebswirtschaftliche Investitionen ${ }^{29}$. Eine steigende Zahl mittelbäuerlicher Betriebe häufte über Jahre hinweg erdrückende Ablieferungsschulden an, die die Eigentümer unversehens zum Spielball politisch motivierter Wirtschaftsstrafverfahren machen konnten. Ablieferungsschulden konnten von den lokalen Behörden leicht politisch instrumentalisiert werden, wenn sie dazu benutzt wurden, mißliebige Dorfmitglieder finanziell zu erledigen, und man beispielsweise Treuhänder einsetzte ${ }^{30}$. Die unbewirtschafteten Flächen umfaßten bis April $1952235000 \mathrm{ha}^{31}$. Sie belasteten die Produktions- und Ernährungsbilanz, bildeten aber zugleich einen Ausgangspunkt, um mittels in politischer Absicht betriebener "Devastierungen“ auf vermeintlich rechtlichem Wege im Frühjahr 1952 die massenhafte Verstaatlichung des Bodens einzuleiten ${ }^{32}$.

Auch die Bestimmungen der DWK über die Düngemittelverteilung fallen in diese erste Gruppe von Maßnahmen. Durch eine Anordnung vom 6. Juli 1949 wurden Düngemittel der plangemäß staatlichen Verteilung unterworfen. Einschneidender war jedoch die "Anordnung über den Verkauf von Düngemitteln an die Bauernwirtschaften", die die DWK am 5. Oktober 1949 erließ. Sie machte die Zuteilung prinzipiell vom Verkauf "freier Spitzen“ an die staatliche Vereinigung der Volkseigenen Erfassungs- und Aufkaufbetriebe (VVEAB) abhängig ${ }^{33}$. Über den verteuerten Verkauf von Düngemitteln, der gekoppelt war an die Erfüllung von Pflichtablieferungsnormen und das Ausmaß der abgelieferten „freien Spitzen“, sollten nicht nur bestimmte, sondern alle landwirtschaftlichen Betriebe zur Produktionssteigerung animiert werden. Die SED-Landwirtschaftsabteilung unter der Leitung von Walter Krebaum war der Motor hierfür; die Skepsis in der Abteilung und aus den Reihen der VdgB blieb unbeachtet ${ }^{34}$. Die Folgen waren verheerend. In Verbindung mit der Hektarveranlagung, die auf eine Erhöhung des Ablieferungssolls hinauslief und die Einnahmen minderte, fehlten vor allem den

28 Die VO „über die Auseinandersetzung bei Besitzwechseln von Bauernwirtschaften aus der Bodenreform" vom 21.6. 1951 regelte in $\$ 1$ Abs. 3, daß die Aufgabe einer Neubauernwirtschaft aus persönlichen Interessen ohne Genehmigung der Kreisbodenkommission unzulässig sei und strafrechtlich als Wirtschaftsvergehen verfolgt werden konnte. Siehe ebd., S. 17.

29 SAPMO NY 4182/900, Bl. 56 f., Kontrollbericht der ZVdgB, 8. 12. 1949. Siehe auch Nehrig, Bauern, S. 236, 241; dies., Uckermärker Bauern, S. 35-40.

30 Werkentin, Strafjustiz, S. 74-78.

31 Schulz, Probleme, S. 53.

32 Dazu aus juristischer Sicht Bell, Enteignungen, S. $49 \mathrm{f}$.

33 ZVOBl. 1949, Teil 1, 26. 9. 1949, S. 721 f., 14. 10. 1949, S. 761 f. Wernet-Tietz, Bauernverband, S. $156 \mathrm{f}$.

34 SAPMO DY 30 IV 2/7/74, Bl. $92 \mathrm{ff}$., Protokoll Abteilungsbesprechung LW der SED, 15. 11.1949. 
klein- und neubäuerlichen Wirtschaften die Gelder zum Ankauf von Dünger. Für die DBD, die wenig später das Landwirtschaftsressort übernahm, war diese Regelung untragbar, weil sie insbesondere ihre Klientel belastete, die kaum „freie Spitzen“" zum höheren Preis verkaufen konnte, vielmehr froh sein mußte, wenn sie ihr Ablieferungssoll erfüllte. Alt- und Großbauern konnten häufig noch von der Substanz zehren und sich den Dünger leisten. Auch wurden sie vielfach durch eingespielte Versorgungswege und Beziehungsnetze in den Dörfern bevorzugt mit Dünger bedient; hilfreich waren auch ihre oft ausgezeichneten Kontakte zu den landwirtschaftlichen Genossenschaften. Diese Strukturen versuchte wiederum die SED auszuschalten ${ }^{35}$.

Während die Düngemittelverordnung nicht primär gegen „Großbauern“ zielte, konnte die Umsetzung der Hektarveranlagung vor Ort häufig mit einem klassenkämpferischen Moment verknüpft werden. Welche Rolle spielte die DBD in dieser Hinsicht? Der Parteivorstand begrüßte die Hektarveranlagung einhellig, liege sie doch im „Interesse der kleinen und mittleren Bauern“ 36 . Nur für diese bäuerliche Gruppe brachte die DBD dann Verbesserungsvorschläge ein. So sollte für sie die geltende generelle Ermäßigung des Ablieferungssolls im Jahr 1949 beibehalten werden. Kinderreiche Familien, die als Selbstversorger einen höheren Eigenbedarf an Lebensmitteln aufwiesen, sollten durch Schutzbestimmungen vor einer ruinösen Ablieferung bewahrt werden. Außerdem empfahl man Kleinbauern die Bildung von Liefergemeinschaften, innerhalb derer sie abwechselnd das Schlachtvieh zur Deckung des Ablieferungssolls aufbringen sollten. Darüber hinaus sprach man sich für eine „Differenzierung“ des Ablieferungssolls zwischen den Ländern und in den Ländern zwischen Kreisen und Gemeinden aus. Darin unterschieden sich die Vorschläge von denjenigen der SED, die nur eine sehr begrenzte Differenzierung für Ausnahmefälle und lokal auf einzelne Betriebe beschränkt vorgesehen hatten ${ }^{37}$. Bezüglich der Hektarveranlagung formulierte man eine Stellungnahme, wonach diejenigen Länder, die wie Mecklenburg und Brandenburg über einen niedrigeren Viehbesatz verfügten, geringer veranlagt werden sollten, was aber unweigerlich zu Lasten anderer Gebiete ging ${ }^{38}$. Die DBD forderte weiter, als Voraussetzung für eine generelle Differenzierung eine einheitliche betriebswirtschaftliche Bewertung der bäuerlichen Wirtschaften zu erstellen ${ }^{39}$.

$35 \mathrm{Vgl}$. Schöne, Genossenschaftswesen in der SBZ/DDR, S. 164-171.

36 ACDP VI-052-0141, Anhang Protokoll PV-Sitzung, 26. 10. 1948 und 1. 11. 1948, auch zum folgenden. Siehe auch die Auflistung von Maßnahmen, verknüpft mit der unbewiesenen These, wonach die DBD zur „Interessenvertretung" der Bauern wurde, bei Reichelt, Blockflöte, S. 48-51.

37 SAPMO DY 30 IV 2/7/170, Bl. 94-96, Aussprache (Merker, Hoernle, Reutter, F. Scholz, Schellenberger) über Hektarveranlagung und Genossenschaftsfragen, 10.11. 1948, hier Bl. 94.

38 ACDP VI-052-0519, Protokoll Sekretariat, 26. 10. 1948.

39 Dabei ging es um Verfahren, den Einheitswert der landwirtschaftlichen Betriebe neu zu erfassen. In diesem Rahmen sollte zur Einordnung der Betriebe ein Kriterienkatalog festgelegt werden, der in umfassender Weise auf betriebswirtschaftlichen Daten basieren sollte; diese erst erlaubten eine Zuordnung zu bestimmten Größenklassen, die über die Höhe der Pflichtablieferung oder etwa auch die jeweiligen MAS-Tarife bestimmten. 
Die DBD-Vorschläge zeichneten sich hier und in den Folgejahren bis $1952 \mathrm{da}-$ durch aus, daß sie die Umsetzungsschwierigkeiten auf der unteren Ebene mitbedachten und unnötige Härten auszugleichen suchten ${ }^{40}$. Die SED hingegen war hieran weitgehend desinteressiert und unsensibel für den Problemdruck, den die verhängten rigiden Maßnahmen erzeugten. Dadurch half die DBD zugleich mit, das Produktionsdiktat mit möglichst geringen Reibungsverlusten durchzuführen, da sie die berechtigten Einwände der Betroffenen geltend machte, solange ihre eigene Nähe zur SED nicht bezweifelt wurde. Eine "Interessenvertretung“ der bäuerlichen Bevölkerung war daher nicht genuines Ziel, sondern hatte sich politischen Prioritäten zu beugen und wurde letztlich dazu eingesetzt, den Erfolg der Landwirtschaftsplanung und -produktion zu garantieren.

In den Jahren 1948/49 hatte die DBD bestimmte Maßnahmen noch öffentlich abgelehnt oder kritische Hinweise vorgebracht, auch um sich coram publico zu profilieren. Mit der zunehmenden inneren Gleichschaltung der Partei verschwand eine solche Vorgehensweise ab 1950. Dies verdeutlicht der Umgang mit der Düngemittelverordnung, die in Kombination mit der Hektarveranlagung den finanziellen Spielraum der Bauern beschnitt. Da die DBD in etwa zeitgleich mit dem Erlaß der Verordnung das Landwirtschaftsministerium übernahm, sah sie sich in einen Begründungszwang gegenüber ihren Mitgliedern gedrängt. Goldenbaum empfahl den Landesvorsitzenden, der Basis unmißverständlich mitzuteilen, daß diese Verordnung ohne das Zutun der DBD entstanden sei. Arthur Pechs Vorpreschen in Brandenburg, der dort als Landesvorsitzender von sich aus eine Änderung angestrebt hatte, wurde jedoch verurteilt, auch um ein Ausscheren einzelner Landesverbände künftig auszuschließen. Statt dessen meldete sich Paul Scholz im „Bauern-Echo“ kritisch zu Wort ${ }^{41}$. Laut internen Ausführungen von Scholz hatte die DBD eine solche Regelung ursprünglich kategorisch abgelehnt und sich daher auch nicht an der Ausarbeitung beteiligt; die von den anderen Parteien erstellte Fassung sei zunächst noch viel schärfer gewesen als die letztlich beschlossene. Denn die DBD hatte noch erreicht, daß Betriebe unter 5 ha NF und solche, die von Schadenskommissionen als bedürftig anerkannt worden waren, von der Regelung ausgenommen wurden. Weitergehende Forderungen der DBD scheiterten daran, daß sie den Brotpreis verteuert oder Preisstützungssubventionen in Höhe von drei Millionen DM erfordert hätten ${ }^{42}$. Die DBD stand in dieser Angelegenheit im Austausch mit den verantwortlichen SED-Stellen, und die von Scholz vorgelegte Linie der DBD wurde wenig später im Ministerrat beschlossen ${ }^{43}$. Sie entlastete die Produzenten durch den zusätzlichen staatlichen Ankauf von „freien

$40 \mathrm{Zu}$ diesem Zweck ging die DBD daran, alle landwirtschaftlichen Kreise anhand eines eigenhändig erstellten Informationssystems zu erfassen. In „Kreisspiegeln“ sollten die Grunddaten festgehalten und mit regelmäßigen Instrukteurberichten gekoppelt werden. Dazu ACDP VI-052-222/1.

41 „Bauern-Echo“, 15. 10., 18. 11. 1949.

42 Zum innerparteilichen Abstimmungsprozeß siehe ACDP VI-052-0143, Protokoll PV-Sitzung, 8. 11. 1949.

43 Zur Modifizierung im Ministerrat: BA DC-20 I/3-1, Ministerratssitzung, 1. 12. 1949, Bl. 134, Vortrag Hamann. 
Spitzen“ an Getreide, der bekanntlich höhere Erlöse als für das Pflichtablieferungssoll einbrachte.

Schließlich hob das Gesetz über die „Maßnahmen zur Erreichung der Friedenshektarerträge" vom 8. Februar 1950 die betriebswirtschaftlich unsinnige Düngemittelrationierung wieder auf, verordnete der Landwirtschaft aber ein ehrgeiziges Ziel, nämlich die Erlangung jener Erträge, die das entsprechende Gebiet 1938 aufgewiesen hatte, bis zum Ende des Zweijahrplanes 195044. Auch 1949 hatte es schon Bemühungen gegeben, die landwirtschaftliche Nutzfläche zu erweitern ${ }^{45}$. Den begrenzten Stellenwert der Landwirtschaft im Zweijahrplan unterstrich die SED-Landwirtschaftsabteilung: „Zu dem Gesetz über die Erreichung der Friedenshektarerträge in der Landwirtschaft ist vorgesehen, in der Einleitung all das aufzuführen, was wir den Bauern bisher gegeben haben, denn wir können der Landwirtschaft nur noch beschränkte Mittel geben. Entscheidend ist jetzt der Aufbau der Schwerindustrie." 46 Durch die Ausdehnung der Anbaufläche sollte langfristig eine Produktionssteigerung und damit die Ernährung auf Dauer gewährleistet werden. Als Anreiz konnten die zusätzlichen Flächen zwei Jahre lang von der Ablieferungspflicht verschont werden, indem man die Anlage von nichtablieferungspflichtigen Grünflächen zuließ. Diese Maßnahme flankierte die angestrebte Erhöhung des Viehbestandes, indem sie auf einen entsprechenden Futteranbau zielte, und verbesserte implizit die Versorgung der Bevölkerung mit Eiweiß.

Initiator dieser Anbauflächenausdehnung war die SKK. In der Abstimmung zwischen SED und SKK fällt auf, wie vehement die SKK dieses Ziel verfocht alternativ wäre eine Mehrproduktion auch durch eine intensivere Bewirtschaftung schon erschlossener Flächen zu erreichen gewesen. Gegen die Flächenausdehnung trugen die SED mit Paul Merker und der DBD-Land wirtschaftsminister Goldenbaum intern bald Zweifel an der Realisierbarkeit dieses Vorhabens vor. Dennoch propagierte Goldenbaum schon im Herbst 1949 das Unternehmen „Friedenshektarerträge" im Parteivorstand und gegenüber den Mitgliedern; Anfang 1950 wurde es in der Funktionärspresse der DBD, im „Pflüger“, vorgestellt ${ }^{47}$.

Die Ministerpräsidenten der Länder mit Ausnahme Thüringens bestärkten Merkers und Goldenbaums Vorbehalte gegenüber einer Politik der Flächenausdehnung. Dies mag dazu beigetragen haben, daß der Gesetzestext die konkret avisierte Zahl von 5088000 ha nicht nannte, die in den Gesprächen mit der SKK

44 Gesetz „zur Erreichung der Friedenshektarerträge“ vom 8. 2. 1950, in: GBl. DDR, 1950, 27. 2. 1950, S. 103-108. Scherstjanoi, „Friedenshektarerträge“, S. 330.

45 SAPMO DY 30 J IV 2/2/18, Protokoll PB, 26. 4. 1949, Bl. 7, Anlage zu VO der DWK über Maßnahmen zur Erweiterung der landwirtschaftlichen Nutzfläche. Das Politbüro forderte Merker zur Überprüfung auf, da der von der DWK beschlossene Verordnungstext von der Politbürofassung abweiche, SAPMO DY 30 J IV 2/2/22, Protokoll PB, 10.5. 1949. Siehe auch SAPMO DY 30 J IV 2/2/26, Protokoll PB, 10. 6. 1949, Bl. 3.

46 SAPMO DY 30 IV 2/7/74, Bl. 92 ff., Protokoll Abteilungsbesprechung, 15. 11. 1949, Zitat Bl. 93.

47 „Bauern-Echo“, 23. 11. und 20. 1. 1949. „Der Pflüger“, Heft 2/1950, S. 5, der Artikel des Wirtschaftsabteilungsleiters beim PV, Keuthe, betonte im Fettdruck: „Es gilt vor allem, die Genossenschaften von alten, reaktionären Einflüssen zu säubern und fortschrittliche, werktätige Bauern in die Vorstände einzubauen.“ 
noch eine zentrale Rolle gespielt hatte. Sie bedeutete eine Ausdehnung der Anbaufläche über den Volkswirtschaftsplan für 1950 hinaus um 78000 ha. Goldenbaum gestand am 3. März 1950 gegenüber dem SKK-Vertreter Kowal ein, daß der

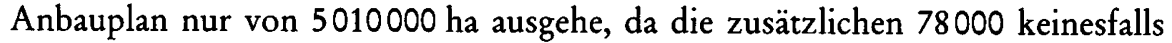
schon im Frühjahr als Ackerfläche in den Anbauplan einfließen könnten. Drei Monate später hielt er es für unmöglich, die Erweiterung $1950 \mathrm{zu}$ erreichen. Schließlich blieb man mit 5004000 Hektar sogar hinter den Vorgaben des Volkswirtschaftsplanes 1950 zurück. Denn die Bauern waren für den arbeits- und geräteintensiven Umbruch der Wiesen und Weiden nicht zu gewinnen ${ }^{48}$.

Bislang ist ungeklärt, warum das Programm der "Friedenshektarerträge“ im Herbst 1950 sang- und klanglos ad acta gelegt wurde; angesichts des Mißerfolgs ist dies jedoch verständlich. Außerdem dürfte das Versagen bei den zuständigen SKK-Vertretern nicht gerade eine positive Einschätzung über Goldenbaum hinterlassen haben. Inwiefern der Fehlschlag auch dazu beitrug, Merker in den Augen der SKK zu diskreditieren, bleibt vorerst noch unklar. Ungeachtet der antisemitischen Motive der SED, die im August 1950 bekanntlich dazu führten, den Nichtjuden Merker wegen seines Einsatzes für die jüdische Minderheit ohne Vorwarnung aus der Partei auszuschließen und bis $1956 \mathrm{zu}$ inhaftieren $^{49}$, verfiel die ZPKK in ihrer Suche nach Belastungsmomenten ausgerechnet auf seine agrarpolitische Arbeit. Dies bot sich aus zweierlei Gründen an: Erstens war es aufgrund der agrarischen Dauerkrise und der unausgereiften Landwirtschaftsverwaltung ein leichtes, Punkte aufzufinden, aus denen sich Vorwürfe zusammenstellen ließen ${ }^{50}$; zweitens dürfte Merkers Image bei den sowjetischen Machthabern in der Tat wegen seiner agrarpolitischen Ansichten und Tätigkeit 1950 gelitten haben.

Kurz nach der Gründung hatte die Parteiführung der DBD auf einer Zonentagung in Weimar am 10. Juli 1948 den Zweijahrplan befürwortet und ihre Unterstützung zugesichert. Die DBD-Presse betonte zum einen in auffälliger Weise die Leistungen der Arbeiter für die Bauern. Außerdem verglich sie den Zweijahrplan in propagandistischer Manier mit dem Marshallplan: Die DBD wies letzteren wie „jeden Plan der Versklavung mit Entrüstung“ zurück, da er die Landwirtschaft nicht voranbringe, sondern „die Tendenz zur extensiven Bewirtschaftung des Bodens" fördere und „sich zum Schaden der kleinen und mittleren Bauern" auswirke ${ }^{51}$. Die Parteiführung war sich auch im klaren darüber, daß ihr Eintreten für den Zweijahrplan im Einheitsfrontausschuß gegen die Einwände der CDU und LDPD ins Spiel gebracht werden würde ${ }^{52}$.

48 Auf Basis sowjetischer Akten: Scherstjanoi, „Friedenshektarerträge“, S. 331-337, zum letzten Punkt anders, S. 328.

49 Siehe dazu Keßler, SED, S. 85-99; Herf, Erinnerung, S. $139 \mathrm{ff}$. und passim, der über weite Strecken eine Merker-Biographie schreibt; ders., Antisemitismus, S. 636-642.

50 Von der ZPKK zusammengestellte Vorwürfe, 11.10. 1950, die sich auf Materialien der ZKSK stützten, SAPMO DY 30 IV 2/4/288, Bl. 1.

51 Siehe Nr. 1 des „Bauern-Echo“, 18. 7. 1948; Artikel zum Zweijahrplan „Vorbildliche Leistung der Arbeiter für die Bauern“. Man begrüßte ausdrücklich die Steigerung der Stickstoff- und Phosphatproduktion. Vgl. auch Wernet-Tietz, Bauernverband, S. $154 \mathrm{f}$.

52 ACDP VI-052-0141, Protokoll PV-Sitzung, 7. 8. 1948, wo klar Paul Scholz, nicht Goldenbaum als Wortführer auftrat und zum Zweijahrplan unterstrich, daß die Mitarbeit der 
Am 5. Oktober 1948 unterbreitete die DBD ihre Vorschläge zum Zweijahrplan. Darin äußerte sie Verbesserungswünsche im Hinblick auf die Anbauplanung - die mehr "Wunschanbauplanung" denn von oben dekretiertes Anbauen sein sollte -, die Betriebsmittelversorgung, die Durchführung des Neubauernbauprogramms nach dem SMAD-Befehl Nr. 209, die Unterstützung für landwirtschaftliche Notstandsgebiete und Stipendien für Schüler in landwirtschaftlichen Ausbildungsstätten ${ }^{53}$. Die Antwort Bruno Leuschners von der DWK desillusionierte die Bauernpartei. Sie lief darauf hinaus, daß die eingeforderten Punkte entweder schon gebührend berücksichtigt oder wenig erfolgversprechend seien. Er sprach explizit der SED-dominierten VdgB entscheidende Aufgaben zu, etwa bei der lokalen Umsetzung der Anbauplanung. Der DBD unterstellte er hingegen, mit der Propagierung von Wunschanbauplänen mehr "die marktwirtschaftlichen Interessen der einzelnen Bauern" statt die „Sicherung der Versorgung" der Konsumenten im Blick zu haben ${ }^{54}$.

Nach dieser Abfuhr gab die DBD nach außen hin nur ihr Engagement für die Verbesserung der Wunschanbauplanung bekannt, nicht ihr Scheitern darin. Damit täuschte sie darüber hinweg, wie sehr Agrarpolitik weiterhin nicht nach landwirtschaftlichen Rationalitätskriterien entschieden wurde, sondern insgesamt der von der SMAD bzw. SKK überwachten Wirtschaftsplanung unterlag; diese räumte dem Aufbau eines industriellen Sektors absoluten Vorrang ein und wertete die Agrarpolitik ideell wie finanziell $\mathrm{ab}^{55}$. Um so fadenscheiniger wirkte Mückenbergers Versprechen gegenüber dem Sekretariat der DBD 1954, wonach die Landwirtschaft einen zentralen Stellenwert im zweiten Fünfjahrplan einnehmen werde, zunächst jedoch erst das „Niveau“ der Industrie erreichen müsse ${ }^{56}$.

Neben Forderungen im Interesse der bäuerlichen Bevölkerung warb die DBDAusarbeitung zum ersten Fünfjahresplan ab 1951 in erster Linie für die in der Wirtschaftsplanung enthaltenen Kontrollen. So sollten Dorfwirtschaftspläne auf der Basis von Dorfspiegeln und Dorfleistungsplänen angelegt werden, die jeden Betrieb erfaßten. Relativen Gestaltungsfreiraum versprachen hingegen die Wunschanbaupläne, die von den Landwirten selbst aufgestellt werden sollten. Eine Reihe von Punkten stützte den staatlichen Sektor der Landwirtschaft und unterschied sich nicht von den Vorhaben der SED: so die DBD-Vorschläge, den MAS zu helfen und die VEG zu Mustergütern auszubauen, ebenso die Bitte an die Agrarwissenschaft, die Lehren von Mitschurin und Lyssenko in der Praxis zu er-

DBD im Block der „Durchführung des Zweijahrplanes und der Festigung der Demokratie zugute" komme.

53 „Bauern-Echo“, 10. 10. 1948, Vorschläge der DBD an die DWK; Reichelt, Blockflöte, S. $50 \mathrm{f}$.

54 BA DC-15/599, Leuschner an Zentral-Vorstand der DBD, 18. 11. 1948, Zitat Bl. 2.

55 Auseinandersetzungen um die Planungskompetenzen führte auch Steidle als stellv. Leiter der HVLF mit der HV Wirtschaftsplanung, in der es eine eigene Abt. Landwirtschaft gab.

BA DC-15/181, Schreiben der Abt. LW in der HV Wirtschaftsplanung, Leuschner, am 27. 4. 1948. Lt. Notiz Bayers am 28. 4. 1948, ebd., habe man Steidle unter Hinweis auf Gespräche mit der SMAD nochmals klargemacht, daß die Sache der Landwirtschaft Angelegenheit der HV Wirtschaftsplanung sei.

56 ACDP VI-052-0526, Protokoll Sekretariat, 15. 3. 1954. 
klären - eine letztlich unlösbare Aufgabe ${ }^{57}$ ! Die in der Industrie erprobten Prinzipien zur Leistungssteigerung sollten auf die Landwirtschaft übertragen werden, wie die Auswertung von Spitzenbetrieben in sogenannten „Beispielwirtschaften“ in jedem Dorf.

Schließlich wiederholte man Anliegen, die teilweise schon im ersten Parteiprogramm angeführt, aber nach wie vor unerfüllt waren: das betraf neben der Wildschweinplage, die enorme Schäden anrichtete, ein einheitliches Steuer- und Beitragswesen und eine Neuregelung der Abgabenordnung zur Sozialversicherung, die Schaffung von Gemeinschaftseinrichtungen, die vor allem Bäuerinnen entlasten sollten, und die "gegenseitige Hilfe" durch Dorfgenossenschaften. Daß die DBD weiterhin am Neubauernbauprogramm festhielt, ist als Reverenz an diese Mitgliedergruppe zu verstehen, denn offiziell war es seit Ende 1949 von der politischen Tagesordnung abgesetzt worden. Die Forderungen zur „Differenzierung“ des Ablieferungssolls und der Absatzregelung trugen eigenständigeren Charakter, da sie kritisierten, beides sei „bisher weniger vom Standpunkt der Landwirtschaft als dem der Versorgung aus gesehen" worden ${ }^{58}$. In den Landessekretariaten erkannten manche sehr wohl, daß die Politik der Wirtschaftsplanung nicht mit einem Konzept der „Dorftumspolitik“ zu vereinbaren war, das die Gründungsfiguren der DBD vertraten und womit sich viele Mitglieder identifizierten ${ }^{59}$.

\section{Ausbau des staatlichen Sektors}

Eine zweite Gruppe agrarpolitischer Maßnahmen der SED zielte auf den Ausbau des staatlichen Sektors. Dieses Gebiet war grundsätzlich nicht als Agenda für die DBD zugelassen, sondern der SED vorbehalten. Anfang 1949 begann der systematische Auf- und Ausbau der MAS, basierend auf den bisher von der VdgB getragenen Maschinenhöfen und den Maschinen-Ausleihstationen, die sich in dieser Form als völlig unzureichend erwiesen hatten, um den Klein- und Neubauern behilflich zu sein 60 . Mit der Gründung der „Verwaltung der Maschinenausleihstationen" (VMAS) im November 1948 verloren die Raiffeisengenossenschaften u. a. ihre Reparaturwerkstätten an die MAS; der Zugriff für „großbäuerliche“ Betriebe auf diese Dienstleistungen wurde dadurch erheblich erschwert. Waren die MAS 1949 noch „staatlich-genossenschaftlich“, also halbstaatlich organisiert, so wurden sie 1950 in eine „staatlich-sozialistische" Organisation überführt. Die VdgB half erheblich mit, den volkseigenen Sektor auszudehnen ${ }^{61}$.

57 Vgl. Fäßler, Freiheit, S. 185-192.

58 Vgl. ACDP VI-052-0143, Ausarbeitung zum Fünfjahrplan bei Protokoll PV-Sitzung, 5. 9. 1950.

59 ACDP VI, unverz. Best. Sekretariatssitzungen der LV, Protokoll Sekretariat Sachsen-Anhalt, 31.5. 1950. Siehe ebd., Protokolle Sekretariat Sachsen-Anhalt, 30. 8. 1949, 9. 12. 1949, 9. 5. 1950.

60 Zur agrarpolitischen Entwicklung 1949 kursorisch Kuntsche, Bauern, S. 233, Nehrig, Landwirtschaftspolitik, S. 297. Siehe SAPMO DY 30 J IV 2/2/4, Protokoll PB, 17. 2. 1949, Bl. 1, 3; Merkel/Schuhans, Agrarwirtschaft, S. 65; Immler, Konzeption, S. 56.

61 Wernet-Tietz, Bauernverband, S. 87; Weißleder, Wesen, S. 93; Schöne, Landwirtschaftli- 
Die Neubauern waren in arbeitstechnischen Fragen weiterhin von der Unterstützung durch die besser ausgestatteten Altbauern abhängig, die sich das Verleihen von Maschinen, Gerät und Gespann oftmals, wie traditionell üblich, in Form von Arbeitsdiensten oder Naturalien entgelten ließen ${ }^{62}$. Ein solches soziales Netz in den Dörfern, das auf gegenseitigen Abhängigkeiten beruhte und sich jenseits verordneter Klassengrenzen und politischer Gesinnung im traditionalen dörflichen Verhaltenskodex bewegte, stimmte nicht mit der Bündniskonzeption der SED überein. Denn die Partei verteilte wirtschaftliche Hilfe und politische Aufwertung nach anderen Kriterien, seitdem sie bewußt nur noch die "werktätige Bauernschaft" als Bündnispartner akzeptierte und allen anderen Teilen der bäuerlichen Bevölkerung die Unterstützung entzog.

Die Vereinigung der Volkseigenen Güter (VVG) wurde zu einer Anstalt des öffentlichen Rechts umgebildet und ihre Verwaltung neu geregelt ${ }^{63}$. Den VEG räumte man bei Veräußerungen von landwirtschaftlichen Privatbetrieben oder Teilen davon ein Vorkaufsrecht ein. Im März 1949 wurde auch die Vereinigung der Volkseigenen Erfassungs- und Aufkaufbetriebe (VVEAB) eingerichtet, die zunächst die Pflichtablieferungsmengen aufkaufte, und mit zunehmender Verdrängung anderer Handelsorganisationen wie $\mathrm{HO}$, Konsumgenossenschaften und ländlicher Genossenschaften aus dem Landhandel bald auch den Aufkauf der „freien Spitzen“ monopolisierte 64 . Das Jahr 1950 bildete eine tiefe Zäsur in der Hinwendung zum staatlichen Sektor. Mit dem „Gesetz über die weitere Verbesserung der Lage der ehemaligen Umsiedler in der DDR“, das am 8. September 1950 in Kraft trat, subventionierte das Bodenreformbauprogramm im wesentlichen nur noch die „Umsiedler" unter den Neubauern ${ }^{65}$. Eine weitere staatlich finanzierte Unterstützung für den Ausbau der kleinbäuerlichen Betriebe außerhalb dieser Gruppe war im Grunde nicht mehr vorgesehen.

\section{Bündnispolitik und „Klassenkampf“}

Eine dritte Gruppe von Maßnahmen stand unter bündnispolitischen wie klassenkämpferischen Vorzeichen. Sie sollte das propagierte Bündnis zwischen SED und Klein- bzw. Mittelbauern unterstreichen, die „Großbauernschaft" aber ausgrenzen. Dazu zählt die offiziell am 20. November 1950 vollzogene Gleichschaltung

ches Genossenschaftswesen, S. 64 f. Zur Verstaatlichung der MAS vgl. Zitat Pieck-Notiz, 18. 12. 1948, oben S. 298.

62 Deutsche Geschichte, Bd. 9, S. 410 f.; Nehrig, Uckermärker Bauern, S. 22 ff., 44-50.

63 Vgl. Deutsche Geschichte, Bd. 9, S. 412. Zur Bildung der VVG und Arbeit am Entwurf siehe SAPMO DY 30 J IV 2/2/11, Bl. 9-11, Anlage 3 zum PB-Protokoll, 21.3. 1949; SAPMO DY 30 J IV 2/2/22, Protokoll PB, 10. 5. 1949, Bl. 3.

$64 \mathrm{Merkel} / \mathrm{Sch}$ hhans, Agrarwirtschaft, S. 48; Tümmler, Agrarpolitik, S. 35; Herzog, Organisationsformen, S. 11.

6522000 Flüchtlingsneubauern verfügten im Oktober 1950 weder über Stall noch Wohnbauten, Bauerkämper, Problemdruck, S. 311; Zank, Wirtschaft, S. 159. 
des ländlichen Genossenschaftswesens ${ }^{66}$. Nachdem die VdgB im Sommer 1948 durch die Bildung eines zentralen Zonenverbandes organisatorisch stabilisiert worden war, betrieben SED-Landwirtschaftsfunktionäre seit Oktober 1948 eine Unterwerfung aller ländlichen Genossenschaften unter die Kuratel der VdgB ${ }^{67}$.

Auch die Bauernpartei war zu diesem Zweck auf die Linie der SED eingeschworen worden. So forderte Goldenbaum Anfang November 1948 im Parteivorstand, „daß die Genossenschaften zu einem vollkommen anderen Kurs kommen müssen", nämlich zur Bildung von einheitlichen Dorfgenossenschaften, auch „Universalgenossenschaften“ genannt, als unterste Basis anstelle diversifizierter Organisationen und einer „Genossenschaftsbürokratie“. Als abschreckendes Beispiel für den letzten Punkt führte er schon 1948 die Rostocker Zentrale der Hauptgenossenschaft Mecklenburgs an, die 1950 in einem Schauprozeß zerschlagen wurde ${ }^{68}$. Die Kursänderung sollte in Zusammenarbeit mit der VdgB realisiert werden, um festzustellen, "wo in den Leitungen der Genossenschaften noch Kräfte sitzen, die in der Vergangenheit Freunde der Junker waren“. Sie müßten rigoros durch Klein- und Mittelbauern ersetzt werden. Weniger den Erlös der bäuerlichen Produzenten als die Interessen der Konsumenten hatte Goldenbaum im Blick, als er für kurze Wege zwischen Erzeugern und Verbrauchern eintrat und dazu anführte, „um so preiswerter erhält der Bauer seine Ware bezahlt“.

Über eine beabsichtigte Stärkung der Rolle der VdgB gegenüber den bestehenden landwirtschaftlichen Genossenschaften berichtete Pieck auch Stalin anläßlich der Moskau-Reise im Dezember 194869. Mit diesem Vorhaben und der Verdrängung angeblich "reaktionärer" Funktionäre in den landwirtschaftlichen Genossenschaften setzte die SED ihre bisherigen Bestrebungen in der Genossenschaftspolitik seit 1945 fort, wobei sie erst im März 1948 von der SMAD aufgefordert worden war, ihren Umgang mit den landwirtschaftlichen Genossenschaften in eine neue politische Konzeption einzufügen ${ }^{70}$. Wie im Herbst 1948 formuliert und auch von der DBD gefordert, betrieb die SED ab Frühjahr 1949 eine Vereinheitlichung der Struktur der landwirtschaftlichen Genossenschaften ${ }^{71}$. Ein Grund hierfür lag darin, daß es ihr bis dahin nicht gelungen war, ihren Mitgliederanteil in

66 Herzog, Organisationsformen, S. 11; Dreesen, Bedeutung, S. 18; Schöne, Landwirtschaftliches Genossenschaftswesen, S. 78-82.

67 SAPMO DY 30 IV 2/7/170, Bl. 94-96, Aussprache über Hektarveranlagung und Genossenschaftsfragen, 10.11. 1948, hier Bl. 95. BA DC-15/737, Bl. 399-415, Vieweg an Rau, 6. 12. 1948. Lt. Bericht fand am 24./25. 11. 1948 eine Aussprache zwischen Vertretern des ZK-Agrarausschusses, den Leitern der Bauernorganisation und der Genossenschaft statt (Bl. 400).

68 ACDP VI-052-0141, Protokoll PV, 1. 11. 1948, Wortbeitrag Goldenbaums zu Genossenschaften, mit Bezug auf Artikel zu Genossenschaftsfragen in „Bauern-Echo“, 19. 10. 1948.

69 Badstübner/Loth (Hg.), Wilhelm Pieck-Aufzeichnungen, S. 250, Antwort auf Fragen zur Besprechung, 18. 12. 1948. Der Bericht Piecks über die Besprechung am 18.12. 1948 vor dem ZS enthielt im verschärften Ton das Vorhaben, die Idw. Genossenschaften von „reaktionären Elementen“ zu säubern. Ebd., S. 268, Bericht Piecks über Besprechung am 18. 12. 1948 vom 27. 12. 1948 vor dem ZS.

$70 \mathrm{Vgl}$. Schöne, Landwirtschaftliches Genossenschaftswesen, S. $41 \mathrm{f}$.

71 Goldenbaum im PV, ACDP VI-052-0141, Protokoll PV, 1. 11. 1948. 
den Genossenschaften zu erhöhen ${ }^{72}$. Offenbar war die Zentralisierung mit einem neuen Konzept der SMAD vom Februar 1949 verknüpft ${ }^{73}$. Anläßlich des Kongresses der landwirtschaftlichen Genossenschaften vom 16. bis 18. März 1949, der unter weitgehender Steuerung der SED ablief, wurden die maßgeblichen Beschlüsse herbeigeführt. Der Plan des Politbüros sah vor, die Genossenschaften zu verpflichten, enger als bisher mit der VdgB, der DLG und dem FDGB zusammenzuarbeiten. Mit dem Argument, daß die Erfüllung des Zweijahrplanes eine zentralere Einbindung erfordere, wurde den regional in Spezialzweige aufgegliederten Genossenschaften eine vereinheitlichte, zentralistische Struktur auferlegt, die auf der unteren Ebene ein Territorialprinzip einführte. Zur Verringerung der Anzahl an Genossenschaften diente die Bildung von Universalgenossenschaften auf Dorf- und Kreisebene - ihre Einführung anstelle der bisherigen landwirtschaftlichen Spezialgenossenschaften hatte das Zentralsekretariat der SED schon im Juli 1948 beschlossen ${ }^{74}$. Die Bildung eines Zentralverbandes landwirtschaftlicher Genossenschaften im Sommer 1949 sollte gegenüber den Ländergenossenschaften eine weitere Zentralisierung bewirken 75 . Prinzipiell war mit dem Zentralverband landwirtschaftlicher Genossenschaften eine Organisation gebildet worden, die mit der Struktur des Genossenschaftswesens in den Westzonen kompatibel gewesen wäre.

In der Bündnispolitik konzentrierte sich die SED sowohl in den landwirtschaftlichen Genossenschaften als auch in der VdgB darauf, den Einfluß von SED-Mitgliedern, meist unter den kleinen und mittleren Bauern, auf Kosten der "großbäuerlichen“ Kreise zu erweitern. Dies sollte vor allem durch die im Frühjahr 1949 anstehenden Wahlen zur Besetzung der VdgB-Orts-, Kreis- und Landesausschüsse - mit mäßigem Erfolg - gelingen ${ }^{76}$. Obwohl der Anteil der SEDMitglieder unter den Gewählten kaum abnahm, analysierte die SED den Ausgang intern als großen Mißerfolg77, hatte sie doch den großen Durchbruch angestrebt.

72 So Schöne, Genossenschaftswesen in der SBZ/DDR, S. 169.

73 Elke Scherstjanoi in einem Vortrag am ZZF, Potsdam, 4. 9. 1995.

74 BA DK-1/8046, Bl. 98-104, Richtlinie zur Arbeit der SED-Mitglieder in ldw. Genossenschaften, am 5.7.1948 vom ZS bestätigt.

75 SAPMO DY 30 IV 2/7/170, Bl. 94-96, Aussprache über Hektarveranlagung und Genossenschaftsfragen, 10.11. 1948, hier Bl. 95; ebd., Bl. 151-156, Bericht über die Sitzung der Genossenschaftskommission der SED, 20.1.1949, bes. TOP 9, Bl. 155. SAPMO DY 30 J IV 2/2/8, Bl. 4-7, Anlage Nr. 1 zum Politbüroprotokoll vom 8. 3. 1949. Siehe dazu auch Wernet-Tietz, Bauernverband, S. 89-92; Herzog, Organisationsformen, S. 9-12; Tümmler, Agrarpolitik, S. 34 f.; Buss, Struktur, S. 45; Schöne, Genossenschaftswesen in der SBZ/ DDR, S. $169 \mathrm{f}$.

76 SAPMO DY 30 IV 2/7/110, Bl. 80-84, Richtlinienentwurf für alle SED-Parteiorganisationen zu den Wahlen, 13.1.1949, gez. Reutter.

77 Ebd., Bl. 108-121, Analyse der Abt. LW, Reutter, über die VdgB-Wahlen 1949 für Merker, 29. 4. 1949. Von 66881 gewählten Mitgliedern der Ortsvereinigungen gehörten $37,4 \%$ der SED an (1947 noch $43 \%)$, $5 \%$ der CDU (1947 auch 5\%), 4,5\% der LDP (1947 noch 4\%), 23 der NDPD, 3,6\% (=3410) der DBD und 49,5\% den parteilosen (1947 48\%). Darin erblickte man zurecht eine von Jahr zu Jahr rückläufige Bewegung der SED-Mitglieder in den Ortsausschüssen der VdgB. Hingegen ist ein klares Übergewicht der SED-Mitglieder zu verzeichnen, je höher die Position im VdgB-Organisationsapparat. Die Vorsitzenden der Ortsvereinigungen: SED 48\%, CDU 5,2\%, LDP 5\%, DBD 4,8\% und Parteilose $37 \%$; 
Die Ressentiments der SED gegen die DBD waren bei der Durchführung dieser Wahlen unübersehbar. Die Bauernpartei hatte erhebliche organisatorische Probleme, ihre Ambitionen in Wahlerfolge umzumünzen. Dort wo die DBD die Bauern für sich mobilisiert hatte, machte ihr die SED den Erfolg oftmals streitig ${ }^{78}$. Auch Eingriffe sowjetischer Kommandanturen zugunsten der SED führten dazu, daß die Wahlen nicht "demokratisch" abliefen, wie der DBD-Landesvorsitzende Martin für Sachsen monierte. Insgesamt warf die ZK-Abteilung Landwirtschaft der DBD vor, sie habe bei den Wahlen "nicht gegen CDU und LDP gearbeitet, sondern gegen die SED“79.

Auch die Auflösung der Tierzuchtämter sollte den "großbäuerlichen“ Einfluß schmälern, verdrängte jedoch ebenso Fachpersonal bürgerlicher Herkunft. Die Viehzucht war in der Tat eine Domäne "großbäuerlicher" Betriebe, weil nur diese bis dahin sowohl Fachkompetenz und Erfahrung über die Qualität des Viehs mitbrachten $^{80}$. Eine späte Entnazifizierung mit dem Ziel des Personalaustauschs zugunsten der SED fand im Zuge der Verwaltungszentralisierung der DWK bei der Bildung der HVLF im April 1948 statt; bis dahin war dort noch eine beträchtliche Zahl früherer Mitarbeiter und Mitglieder des Reichsnährstands tätig81. 1950 erreichten die personellen "Säuberungen“ eine neue Dimension. Daraus erwuchsen besondere kaderpolitische Aufgaben für die $\mathrm{DBD}$, die die entstehenden Lücken durch fachlich wie politisch im Sinne der SED geschultes Personal auffüllen sollte. Im gleichen Jahr griff die SED mit Unterstützung der ZKSK zu härteren Mitteln

noch deutlicher die Verteilung bei den insgesamt 128 Vorsitzenden der Kreisausschüsse: SED 106, CDU keiner, LDP 3, DBD 15 und Parteilose 4.

78 ACDP VI-052-0141, Protokoll PV-Sitzung, 7. 4. 1949, besonders die Ausführungen Martins für Sachsen und Sejnas für Sachsen-Anhalt monierten, daß man die DBD bei Kandidatenlistenplätzen betrogen habe. In Sachsen-Anhalt hätten sich daher die Bauern aus der DBD mit jenen aus bürgerlichen Parteien gegen die SED zusammengetan. Dem PV-Protokoll zufolge lautete das Ergebnis offenbar der gewählten Mitglieder in den Ortsvereinigungen zu diesem Zeitpunkt nach DBD-Angaben: 54\% SED, 18,1\% parteilos, $13 \%$ DBD, 7,4\% CDU, 6,8\% LDP und 0,3\% NDPD; es weicht signifikant zugunsten von SED und DBD und zu Lasten der Parteilosen von Reutters Analyse oben ab. Zu Organisationsproblemen der DBD in Thüringen, SAPMO DY 30 IV 2/7/277, Bl. 204, LV SED Thüringen, Abt. LW an ZS, 12. 4. 1949. Für Sachsen vgl. Fritsch, Verwirklichung, S. 146$149,167$.

79 SAPMO DY 30 IV 2/7/63, Bl. 58 ff., Protokoll Abteilungssitzung LW, 20. 4. 1949, Zitat Bl. 59. Außerdem stellte man fest, daß in der Hälfte aller SED-Parteiorganisationen keine Bauern organisiert sind.

80 ACDP VI-052-0141, Protokoll PV-Sitzung, 26. 8. 1948.

81 Im Frühjahr 1948 kam es zu einer Entlassungswelle in der HVLF, die vor allem ehemalige Mitglieder der NSDAP und ihrer Organisationen sowie während der NS-Herrschaft tätige staatliche und kommunale Bedienstete traf. Unter den Neueinstellungen zum 1.4. 1948 überwogen die SED-Mitglieder. Im Februar 1949 zählte die HVLF noch 23 Angestellte, die der NSDAP oder einer ihrer Gliederungen angehört hatten. Siehe dazu BA DK-1/ 2046, Bl. 109 [194 zweifach paginiert], Bl. 115 [200]), Bl. 131[217]-148[234] Auflistung vom 17. 12. 1948. Von 320 Mitarbeitern der HV gehörten 104 der SED an, 6 der CDU, 3 der LDP, niemand der DBD oder NDPD, 207 waren parteilos. Das Ministerium wurde lt. Kramer, selbst von der „Säuberung“ erfaßt, 1949 mit linientreuen Kommunisten besetzt; Kramer, Bolschewisierung, S. 116 f.; vgl. Bauerkämper, Wege, S. 258 f.; zur DWK 1947/48: Steiner, Deutsche Wirtschaftskommission. 
und inszenierte in Mecklenburg zwei große Schauprozesse. Nahezu alle leitenden Genossenschaftsfunktionäre der Raiffeisengenossenschaft Mecklenburgs waren von den Prozessen in Güstrow (10. bis 15. Juli 1950) und in Greifswald (16. bis 18. Juli 1950) betroffen ${ }^{82}$.

Trotz der „Säuberungs“-Politik der DBD 1950 in ihren eigenen Reihen zeichnete sie sich im Vergleich zur SED durch einen moderateren "Großbauern“-Begriff aus. Gerade angesichts der Unschärfe der Definition von 1950, wie sie offensichtlich die SKK vertrat, gerieten in diesem Jahr auch vormals als mittelbäuerlich klassifizierte Betriebe in die Schußlinie. Schon Höfe ab 10 ha LNF konnten unter gewissen Umständen zu den "großbäuerlichen" zählen ${ }^{83}$. So ist es nicht verwunderlich, daß sich auf dieser Berechnungsgrundlage in der Rezeption von SED und SKK eine Übermacht der „Großbauern“ abzeichnete, die demnach ein Viertel der bäuerlichen Betriebe innehatten und drei Mio. ha Land besaßen, während 260000 Kleinbauern 530000 ha und 251000 mittlere Betriebe 1991000 ha ihr Eigentum nannten. Nur kleine und mittlere Betriebe bis 10 ha LNF sollten noch wirtschaftlich entlastet werden. Die Erfassungs- und Aufkaufpolitik für 1950 sah 500 Millionen DM weniger Einnahmen bei gleicher Ablieferungsmenge für die landwirtschaftlichen Betriebe vor, weil zwar die Erfassungspreise für das Soll erhöht, die Aufkaufpreise für die „freien Spitzen“ aber drastisch gesenkt werden sollten ${ }^{84}$. Denn die Einkommensschere zwischen den "Großbauern" und den Arbeitern klaffte nach Ansicht von SED und SKK weit auseinander, das Einkommen eines "Großbauern" sei fünfmal so groß wie das eines Arbeiters.

Weite Teile der Bauernschaft, nachweislich in Sachsen und Sachsen-Anhalt, die häufig der CDU nahestanden, vollzogen die ideologisch motivierte Abtrennung der "Großbauern“ aus dem traditionellen dörflichen Milieu nicht mit und verweigerten die angeordnete soziale Aufspaltung in den Dörfern. Bisweilen wollten Klein- und Mittelbauern im Jahr 1950 die gestiegenen Ablieferungslasten für die "Großbauern" mitübernehmen, und Neubauern forderten die Wiedereinsetzung der vorherigen Besitzer ${ }^{85}$. In der Großbauern-Frage verlangte die DBD-Führung - auf Veranlassung der SKK - ihren Funktionären und Mitgliedern eine nicht ohne weiteres nachvollziehbare Strategie ab. „Großbauern“" waren als einfache Mitglieder, sofern sie der Partei bereits angehörten, geduldet, durften aber künftig nicht mehr aufgenommen werden. Mit erheblichem Aufwand versuchte die Parteiführung, sie aus den Leitungen zu verdrängen. Hingegen sollten sie im Hinblick auf die Deutschlandpolitik durch die DBD integriert und im Rahmen eines

82 Wernet-Tietz, Bauernverband, S. 91 f., Buss, Struktur, S. 70-76, Bauerkämper, Führungsgruppen, S. 86-88.

83 Badstübner/Loth (Hg.), Wilhelm Pieck-Aufzeichnungen, S. 337-339, Besprechung am 23. 3. 1950 mit Tschuikow, Iljitschow, Rau, Grotewohl und Pieck. Laut einem Bericht über die Parteiarbeit auf dem Lande, höchstwahrscheinlich von Juni 1950, zählte man einen Teil der Bauern von 15-20 ha zu den Großbauern; SAPMO DY 30 IV 2/7/243, Bl. 26-33.

84 Badstübner/Loth (Hg.), Wilhelm Pieck-Aufzeichnungen, S. 337-339, Besprechung am 23. 3. 1950, S. 337.

85 ACDP VI-052-0143, Protokoll PV, 17. 6. 1950, Ausführungen Martin, Sachsen; ACDP VI-052-0522, Protokoll Sekretariat, 16. 5. 1952. 
Engagements in der "Nationalen Front" mobilisiert werden ${ }^{86}$. Diese ideologischen Feinheiten im alltäglichen Umgang waren für die normalen Mitglieder, die zu dieser Zeit meist keine politische Schulung durchlaufen hatten, kaum einsichtig.

\section{Künftiger Bündnispartner? Das Beispiel des Landarbeiterschutzgesetzes}

Im Hinblick auf die Landarbeiterschaft zielten die sowjetischen Vorgaben darauf $a b$, langfristig die sozialistische Entwicklung auf dem Land zu begünstigen - und unter kurzfristigem Blickwinkel die Landarbeiterschaft in das politische Lager der SED zu integrieren ${ }^{87}$. Die sozialpolitische Strategie gegenüber den Landarbeitern, deren Lage verbessert und damit an das höhere Niveau der selbständigen Kleinund Mittelbauern angeglichen werden sollte, ließ sich mit einem agrarmarxistischem Ideologem zur Gründung der LPG verknüpfen ${ }^{88}$. Schon im Vorfeld offener Kollektivierungspolitik galt es, Unterschiede zwischen den sozialen Schichten der potentiellen Genossenschaftsmitglieder einzuebnen. Die Entstehung des Landarbeiterschutzgesetzes zeigt exemplarisch das Dilemma der DBD im politischen Entscheidungsprozeß und die innerparteilichen Reaktionen auf verschiedenen Ebenen. Das Dilemma ging auf die weitgehende Steuerung des Gesetzgebungsverfahrens durch sowjetische Stellen und die Umsetzung durch die SED zurück, beide Seiten engten die DBD ein.

Das am 1. Januar 1950 in Kraft tretende „Gesetz zum Schutze der Arbeitskraft der in der Landwirtschaft Beschäftigten "89 sollte eine eklatante arbeitsrechtliche Lücke schließen. Die sogenannte „Landarbeiterschutzverordnung“ löste eine seit Januar 1919 geltende „vorläufige Landarbeitsordnung“ ab, die wiederum die überkommene Gesindeordnung und die diskriminierenden Ausnahmegesetze gegen Landarbeiter aufgehoben und die Arbeitszeiten begrenzt hatte ${ }^{90}$. Insgesamt war das Gesetz Teil jenes Projekts, das die Eigentümergesellschaft allmählich in eine Arbeitnehmergesellschaft zu transformieren suchte, und das die anfangs privatbäuerlich geprägte Agrarstruktur bis 1963 grundlegend umwälzte. Die unmittelbare Vorgeschichte des Landarbeiterschutzgesetzes begann im Dezember 1948. Welch akuter Handlungsbedarf bestand, war schon seit Jahren offensichtlich"1.

86 ACDP VI-052-0143, Protokoll PV, 27./28. 2. 1950, S. 24f. ACDP VI-052-0143, Protokoll PV, zugleich Festsitzung anläßlich des zweijährigen Bestehens, 16. 6. 1950, Ausführungen Goldenbaum mit Bezug auf GAK und Gehricke.

87 Beispielhaft BA DK-1/8114, Bl. 149ff., Bericht MLF Sachsen an HVLF, 4. 2. 1949, hier Bl. 162.

88 Siehe dazu Bergmann, Probleme, S. 75; Gey/Quaisser, Konzeptionen, S. 19ff.; vgl. zur Landarbeiterschaft bis 1960 auch Bauerkämper, Aufwertung, S. $266 \mathrm{f}$.

89 GBl. DDR 1949, Nr. 16, 19. 12. 1949, S. 113 f.

90 Winkler, Revolution, S. 87 f. SAPMO DY 30 J IV 2/2/11, Bl. 13-16, $\$ 18$ Anlage 5 des Protokolls der PB-Sitzung, 21. 3. 1949, Bl. 16.

91 Entsprechend emphatisch wurde das Gesetz in der DDR als Errungenschaft gefeiert; Frerich/Frey, Sozialpolitik, S. 116 mit Verweisen auf DDR-Literatur. 
Die Landarbeiter distanzierten sich klarer noch als die Bauernschaft von der SED, obwohl sie doch als Teilgruppe des Proletariats in ideologischer Nähe zur Partei der Arbeiterklasse hätten stehen müssen. Die SED hatte deren politische Einbindung versäumt.

Die Arbeitsbedingungen auf dem Land hinkten in der Tat denen in der Industrie hinterher, solange es allerdings um die Ernährungssicherung ging, bot eine Beschäftigung auf dem Land einen entscheidenden Vorteil in der von Mangelernährung geprägten Zusammenbruchsgesellschaft der unmittelbaren Nachkriegszeit'22. Dieser ernährungswirtschaftliche Aspekt dürfte teilweise erklären, warum eine Neuregelung nicht früher erfolgte. Denn die Verordnung verschlechterte die Produktionsbedingungen erheblich und gefährdete den agrarwirtschaftlichen Erfolg vieler Betriebe aller Größengruppen. Daher ist eine wirtschaftliche Motivierung des Gesetzes, wonach es einen finanziellen Anreiz für mehr und bessere Landarbeit schuf, äußerst zweifelhaft ${ }^{93}$.

Während die SED im Mai 1949104705 von insgesamt rund 950000 Bauern als Mitglieder und Kandidaten in ihren Reihen zählte, gewann sie nur einen geringeren Teil der Landarbeiter. Von rund einer Million Land- und Forstarbeitern 1949/ 50 gehörten im Mai 194962421 der SED als Mitglied oder Kandidat an ${ }^{94}$. Die Mitgliederanteile differierten je nach wirtschaftlicher Struktur der Länder erheblich. Während im Oktober 1948 in Sachsen der Anteil der Bauern an der SED-Gesamtmitgliederzahl bei nur 2,5\% lag und der der Landarbeiter sogar bei nur 1,9\%, betrug zum gleichen Zeitpunkt in Mecklenburg der der Bauern 15,3\% und der der Landarbeiter 3\%. Den vergleichsweise höchsten Landarbeiteranteil zählte die SED in Sachsen-Anhalt mit 5,4\%, der Anteil der Bauern lag hier nur um 1\% höher'55.

Nur knapp ein Fünftel der Landarbeiter war bis März 1947 gewerkschaftlich organisiert, regional lagen die Sätze erheblich darunter ${ }^{96}$. Im Herbst 1948 gehörten nicht mehr als 20\% der 179078 Landarbeiter Mecklenburgs dem FDGB an, dagegen gut zwei Drittel der Forstarbeiter ${ }^{97}$. Immerhin verbuchte die Gewerkschaft Land und Forst bis Dezember 1949 einen Mitgliederanstieg auf 428000, verfügte dann 1953, vermutlich infolge des allgemeinen Rückganges der Land-

92 Die Behörden gingen davon aus, daß die Landarbeiter schon mitversorgt werden würden, sofern sie nicht selbst dazu in der Lage wären: Vgl. BA DC-15/715, Bl. 18, Kowal, Chef der SMAD-Abt. LW, an Rau, 14. 5. 1948; Rau sprach sich dafür aus, auch die Landarbeiter mit Lebensmittelkarten auszustatten; Kowal war nur in absoluten Ausnahmefällen dazu bereit, ansonsten gehörten ldw. Arbeiter und ihre Familien zu dem sich voll selbstversorgenden Teil der Bevölkerung.

93 So lautete Dillwitz zufolge die Einschätzung von Merker, Hoernle (HVLF) und Brack (Abt. Arbeit und Sozialfürsorge). Dillwitz, Entwicklung, S. 61.

94 SAPMO DY 30 IV 2/7/243, Bericht über die Parteiarbeit auf dem Lande, undatiert [1950], Bl. 26-58, hier Bl. 26f.; Zahlen für Mai 1947 bei Müller, Sozialistische Einheitspartei Deutschlands, S. 510: 64276 (3,6\% der SED-Mitglieder).

95 Zahlen für Oktober 1948 nach: Entscheidungen, Anhang, Tabelle 8.

96 Dillwitz, Entwicklung, S. 55.

97 Woderich, Zu den Anfängen, S. 55f. Auffälligerweise waren die Forstarbeiterinnen sogar zu $95 \%$ organisiert, die Landarbeiterinnen hingegen nur zu $12 \%$. 
arbeiterschaft, noch über 320000 Mitglieder ${ }^{98}$. Im Jahr 1949 gab es etwa 910000 Landarbeiter und zeitweilige Lohnarbeitskräfte, wovon 832000 in der privaten Landwirtschaft beschäftigt waren. Fast ein Drittel der in Privatbetrieben tätigen familienfremden Arbeitskräfte war auf „großbäuerlichen“ Höfen mit über 20 ha beschäftigt; berücksichtigt man nur die Landarbeiter und klammert Saisonarbeitskräfte aus, so arbeiteten $42 \%$ von ihnen bei „Großbauern“99. Das war zwar eine erhebliche Zahl, sie bedeutete jedoch zugleich, daß mehr als die Hälfte der Landarbeiter in den anderen Betriebsgrößenklassen tätig war. Damit war auch die Zielgruppe der DBD, die Klein- und vor allem die Mittelbauern, erheblich von der Landarbeiterschutzverordnung betroffen.

Zwar registrierte die SED ebenso Unzulänglichkeiten ihrer Politik auf dem Land, doch sie ergriff keine Initiative, die darauf zielte, die Landarbeiter stärker an sich zu binden. Die bisher zugänglichen Dokumente zur Reise der SED-Führungsspitze nach Moskau vom 12. bis 24. Dezember 1948 enthalten eindeutige Indizien, wonach der Anstoß zum Landarbeiterschutzgesetz von sowjetischer Seite ausging. Es gibt keinerlei Hinweise dafür, daß eine solche Gesetzesinitiative ursprünglich von seiten der SED vertreten wurde ${ }^{100}$, obgleich es durchaus Anknüpfungspunkte an kommunistische Forderungen für die Landarbeiterschaft aus der Weimarer Zeit gegeben hätte ${ }^{101}$. Insofern trägt die sofortige Ingangsetzung der Gesetzesvorbereitung Züge eines sowjetischen „Re-Imports“ ursprünglich deutscher Traditionsbestände im Sozialversicherungswesen ${ }^{102}$.

Im Frühjahr 1949 begannen die Vorarbeiten in verschiedenen Abteilungen des Parteivorstandes der SED zu einer Reihe von agrarpolitischen Gesetzesvorhaben. Während der DWK die Verordnungen ernährungstechnischer Art überlassen

98 Zahlen für 1949 nach Müller, FDGB, in: SBZ-Handbuch S. 660, für 1953 nach Schulz, Probleme, S. 157. Dennoch bewiesen die Landarbeiter 1953 ihre Distanz. Nach dem 17. Juni führte widersetzliches Verhalten dazu, daß die laufenden Gewerkschaftswahlen der IG Land und Forst unterbrochen werden mußten, ebd.

99 Schulz, Probleme, S. 6; vgl. auch Bauerkämper, Von der Bodenreform, S. 128-131.

100 Siehe Piecks „Notizen für die Besprechung in Moskau“, die dann am 18.12. 1948 stattfand, in: Badstübner/Loth (Hg.), Wilhelm Pieck-Aufzeichnungen, S. 254-259, hier S. 255. Auffälligerweise enthalten weder diese einen Hinweis auf ein von der SED geplantes Landarbeiterschutzgesetz noch eine ausführlichere Ausarbeitung „Antwort auf die Fragen zur Besprechung“, ebd. S. 247-253, die den Herausgebern zufolge vorher dem Politbüro zugeleitet worden war, also nur die Pläne der SED enthielt, ohne die späteren, in Moskau besprochenen Änderungen. Dagegen enthalten die Notizen Piecks, die er vermutlich noch in Moskau angefertigt hatte, und die ihm erheblich erscheinenden Ergebnisse der Beratungen und damit auch die Kurskorrekturen Stalins festhielten, erstmals und eindeutig den Hinweis auf ein "Gesetz über Landarbeiter"; ebd., Ergebnis der 4stündigen Besprechung am 18. 12. 1948, S. 259-263, Zitat S. 260. So auch die Notizen Piecks für den Bericht vor dem ZS über die Besprechungen in Moskau, ebd., S. 265-274, bes. S. 268. Nicht umsonst griff der PV der DBD in einem Schreiben an die SKK (Buschmanow) gezielt das Landarbeiterschutzgesetz heraus, um auf weitere „Einsatzmöglichkeiten“ der DBD zu verweisen. ACDP VI-052-0001c, Stellungnahme zu einigen politischen Problemen lt. Schreiben vom 30. 5. 1950. Unzutreffend Dillwitz, Entwicklung, S. 60.

101 Siehe etwa die kommunistische Landtagsfraktion in Mecklenburg-Schwerin 1926-29, Für die Arbeiter- und Bauernmacht, S. $51 \mathrm{ff}$., $58 \mathrm{ff}$., $65 \mathrm{ff}$. Für die Tolerierung einer SPDRegierung rangierten Landarbeiterfragen ganz oben.

102 Hockerts, Einführung, S. 9. 
wurden, konzentrierte sich die SED auf die Erweiterung des „volkseigenen“ Sektors in der Landwirtschaft. Eine Bauernkonferenz der SED in Halle diente der zusätzlichen - vordergründig basisnahen - Scheinlegitimation ${ }^{103}$. Die SED-Landwirtschaftsabteilung erstellte in engster Zusammarbeit mit der SMAD Entwürfe ${ }^{104}$. Bereits im März 1949 beschloß das Politbüro eine Vorlage, die zwar wesentliche Punkte des späteren Gesetzes schon enthielt, in der aber auffälligerweise manche begriffliche und inhaltliche Schärfen noch fehlten, über die die SED später mit CDU und LDP vehement stritt; so z.B. die Zuspitzung der Verordnung auf "private und diesen verwandte" landwirtschaftliche Betriebe ${ }^{105}$. Während die frühe Politbürovorlage explizit die Beschäftigten in den VEG miteinbezog, ist von diesen später nicht mehr ausdrücklich die Rede, sie wurden unter die Bezeichnung "verwandte" Betriebe subsumiert. Diese Veränderung dürfte auf Veranlassung sowjetischer Stellen nachträglich eingebaut worden sein, um die Zielrichtung des Gesetzes gegen all jene privatbäuerlichen Betriebe, die Landarbeiter beschäftigten, zu unterstreichen, denn diese zählten laut Doktrin eindeutig nicht mehr zur Kleinbauernschaft ${ }^{106}$.

Auch in der SED setzte zu dieser Zeit eine Tendenz ein, grundsätzlich zwischen Kleinbauern, die Landarbeiter beschäftigten und solchen, die das nicht taten, zu unterscheiden. Das Landarbeiterschutzgesetz markierte damit eine Radikalisierung in der "Bündnispolitik“, die alle Bauern, die fremde Arbeitskräfte beschäftigten, ob Klein-, Mittel- oder Großbauern, als opportune Bündnispartner der SED in Frage stellte. Trotz der Verhandlungen in der DWK - dort besprach man offensichtlich sieben verschiedene Vorlagen des Gesetzes ${ }^{107}$ - und mit den bürgerlichen Blockparteien, trotz der Anhörung von VdgB und DBD beweist die Aktenüberlieferung, daß die Eckpunkte von den SED-Fachabteilungen gemeinsam mit der SMAD festgelegt worden waren ${ }^{108}$.

Welche Punkte sah nun das Landarbeiterschutzgesetz nach der in der DWK besprochenen, maßgeblichen Fassung vor? Zunächst führte es schon nach kurzer Beschäftigungsdauer das Prinzip eines festen schriftlichen Arbeitsvertrages ein, der von der zuständigen Industriegewerkschaft (IG) Land und Forst - letztlich auf Kosten der Arbeitgeber - zu registrieren war und so auf seine Zulässigkeit hin

103 Siehe Deutsche Geschichte, Bd. 9, S. 412.

104 Absprachen zum Landarbeiterschutzgesetz in SAPMO DY 30 IV 2/7/74, B1. $19 \mathrm{ff}$., Abteilungsleiterbesprechung, 31.1.1949.

105 Siehe dazu SAPMO DY 30 J IV 2/2/11, BI. 13-16, Anlage 5 zum Protokoll der PB-Sitzung vom 21.3. 1949 und SAPMO DY 30 IV 2/7/110, Bl. 130-136, Text der VO zum Schutz der Arbeitskraft der Landarbeiter. Das Sekretariat der DWK bereitete für die Vollversammlung der DWK am 28./29. 7. 1949 eine erneut veränderte Fassung vor, siehe SAPMO NY 4090/325, BI. 139-142.

106 Allerdings ging die Bezeichnung „landwirtschaftlicher Unternehmer“ und „Beschäftigter" auf eine Angleichung der Begrifflichkeit in den parallel ausgearbeiteten Tarifverträgen für die private Landwirtschaft zurück. SAPMO DY 30 IV 2/7/113, Bl. 32, Reutter an Pfaffrath, Abt. Arbeit und Sozialfürsorge, 10. 5. 1949.

107 So Otto Körting im Rückblick, SAPMO VdgB 144, Protokoll Landesausschußsitzung Sachsen-Anhalt, 18. 7. 1950.

108 SAPMO DY 30 IV 2/7/113, Bl. 32, Reutter an Pfaffrath, Abt. Arbeit und Sozialfürsorge, 10. 5.1949. 
überprüft werden konnte. Dieser Vertrag sollte verbindliche Festlegungen zur Art der zu verrichtenden Arbeit enthalten. Die Arbeitszeit sollte prinzipiell acht Stunden täglich oder 48 Stunden wöchentlich betragen, worin die Zeiten für die Viehfütterung und Pflege inbegriffen waren - eine nicht selbstverständliche Einbindung. Der Acht-Stunden-Tag war ein Kernstück der Verordnung. Die Verträge sollten die Entlohnung nach Einstufung in einen Tarifvertrag regeln, fixierten einen Urlaubsanspruch sowie Zahlungen bei Krankheit und Tod. Außerdem enthielten sie Abmachungen zur Unterbringung und zur kostengünstigen Lebensmittelversorgung der Landarbeiter. Verheirateten Landarbeitern war eine angemessene, nötigenfalls möblierte Wohnung, eventuell auch ein Stall und Kartoffelland bereitzustellen, ledige Landarbeiter hatten Anspruch auf ein möbliertes beheizbares Zimmer ${ }^{109}$.

In Kombination mit den parallel dazu ausgearbeiteten Tarifverträgen für die Landarbeiter in privaten Betrieben, die ab April 1949 in Kraft traten ${ }^{110}$, bedeutete das Gesetz eine erhebliche finanzielle, soziale und rechtliche Verbesserung für die Landarbeiterschaft und gleichzeitig eine nicht weniger einschneidende Belastung für die Arbeitgeber. Letzteren drohte das Gesetz bei Zuwiderhandlung eine Geldstrafe bis zu 5000 DM und Gefängnis bis zu sechs Monaten an ${ }^{111}$.

Das Landarbeiterschutzgesetz erstrebte eine Angleichung der agrarischen Arbeitsverhältnisse an die städtisch-industriellen; das Wohlstandsgefälle zur Industriearbeiterschaft sollte vermindert werden. Normativ überprüf- und einklagbare vertragsrechtliche Regelungen sollten gewohnheitsrechtliche ablösen. Der bislang nicht vorhandene Zugriff der Gewerkschaften auf die bäuerlichen Betriebe sollte verstärkt werden. So hatte die IG Land und Forst faktisch ein Vetorecht bei Entlassungen, denn jede Kündigung bedurfte ihrer Zustimmung, und sie mußte bei Arbeitsstreitigkeiten eingeschaltet werden ${ }^{112}$. Das Landarbeiterschutzgesetz stand in Zusammenhang mit der Zielrichtung der Wirtschaftspolitik für das Jahr 1950, die einen verschärften Ausbau der Schwerindustrie vorsah und die Agrarpolitik diesem Kurs unterordnete. Dabei wurde inoffiziell eingefordert, daß die Landwirtschaft einen Tribut zum industriellen Aufbau leisten und auf RessortMittel verzichten müsse ${ }^{113}$. Die verteuerte ländliche Arbeitskraft führte zu einer Abwanderung in industrielle Wirtschaftszweige, weil sich viele bäuerliche Betriebe die Beschäftigung von familienfremden Arbeitskräften zu diesen Konditio-

109 GBl. DDR 1949, Nr. 16; SAPMO DY 30 IV 2/7/110, BI. 130-136.

$110 \mathrm{Vgl}$. grundsätzlich Dillwitz, Entwicklung, S. $52 \mathrm{ff}$.

111 Kriminalisiert wurden Arbeitgeber, die Landarbeiter ohne Verträge beschäftigten, wer unter Tarif entlohnte, die gesetzliche Arbeitszeit um mehr als 2 Stunden überschritt oder es unterließ, den Arbeitsvertrag bei der IG Land und Forst registrieren zu lassen. Siehe $\$ 11$ der Verordnung. Der DWK-Entwurf sah noch 10000 DM Strafe vor.

112 SAPMO NY 4090/325, Bl. 140, $\$ 2$, Abs. 5, \$9, Beilegung von Arbeitsstreitigkeiten. Offenbar gab es gewerkschaftliche Vorbehalte gegen die Übernahme der Registrierung der Arbeitsverträge durch die IG Land und Forst; man argumentierte, daß damit eine staatliche Aufgabe übernommen werde, die den Charakter der Arbeitnehmervertretung verwische und daher besser bei den Arbeitsämtern angesiedelt werden sollte. Dies griff Merker auf in SAPMO NY 4102/49, Bl. 185-189, Zeitungsbeitrag für das „Neue Deutschland“ „Noch einmal Landarbeiterschutzgesetz", 21. 7. 1949.

113 Vgl. Nehrig, Bauern, S. 241. 
nen nicht mehr leisten konnten. Die Auswirkungen des Gesetzes, ob intendiert oder nicht, deckten sich daher mit der in Angriff genommenen Arbeitskräftelenkung in jene Wirtschaftszweige, die den industriellen Aufbau vorantrieben ${ }^{114}$.

In der Praxis bedeuteten die im Entwurf vorgesehenen Regelungen erhebliche Erschwernisse für die bäuerlichen Wirtschaften. In den Beratungen bei der DWK mit allen Parteien und der VdgB sind daher viele Einwendungen vorgebracht worden. Offenbar hielten sich CDU und LDP in ihrer offiziell geäußerten Kritik zurück. Sie kritisierten die Verordnung nicht prinzipiell, sondern in ihrer Ausrichtung auf die "privaten " bäuerlichen Betriebe und in einigen anderen Details ${ }^{115}$. Allerdings verfolgten ihre Verhandlungsvertreter nach der Staatsgründung die Devise, in Parlamentsausschüssen wesentliche Nachbesserungen zu erreichen. Die Fraktionsmitglieder von CDU und LDP verfochten hierbei anscheinend einen Kurs, der von jenem, den die jeweiligen Parteiführungsgremien nach außen hin vertraten, abwich ${ }^{116}$. In Sachsen blieb der CDU-Vertreter im Landesblock der Sitzung, die das Landarbeiterschutzgesetz behandelte, fern ${ }^{117}$.

Während sich VdgB und DBD offiziell ganz hinter die Verordnung stellten, entzündete sich in beiden Organisationen ein Meinungsstreit, der im internen Machtkampf instrumentalisiert wurde. Der erste Vorsitzende der ZVdgB, Otto Körting (SED), wandte sich gegen eine Reihe von Punkten, die seiner Auffassung nach erhebliche finanzielle Belastungen für die ohnehin wirtschaftlich instabilen bäuerlichen Betriebe mit sich bringen würden ${ }^{118}$. Seine Argumentation, derzufolge die Bauern allein bei der VdgB mit 35 Mio. DM verschuldet und teilweise nicht einmal das ihnen zustehende Düngerkontingent kaufen könnten, brachte ihn in Verdacht, eine "Bauernpolitik“ [!] anstelle einer politisch konformen Agrarpolitik zu verfolgen ${ }^{11}$. Es war verhängnisvoll, daß diese Äußerungen über den internen Rahmen der Beratungen zum Landarbeiterschutzgesetz in der DWK hinaus bei einer interfraktionellen Sitzung der Volkskammer fielen. Die SED warf ihm neben anderen Punkten vor, von der bündnispolitischen Linie „abgewichen“

114 Zur Arbeitskräftelenkung vgl. auch das „Gesetz der Arbeit“ vom 19. 4. 1950, GBI. DDR 1950, Nr. 46, 28. 4. 1950, S. 352. Dazu auch Wunderlich, Farmer, S. 62 f. Eine andere Einschätzung bei Bauerkämper, Von der Bodenreform, S. 131; demzufolge konnte das Landarbeiterschutzgesetz die Abwanderung Erwerbstätiger in andere Bereiche nicht aufhalten, wobei mitschwingt, als habe das Gesetz eine solche verhindern wollen.

115 BA DC-15/826, Abänderungsvorschläge der Parteien und Organisationen zum Entwurf der VO zum Schutze der Arbeitskraft der Landarbeiter, undatiert [20. 7. bis 11. 10. 1949]. CDU und LDP traten für die Halbierung des Strafmaßes ein und konnten dies durchsetzen. Vgl. auch Dillwitz, Entwicklung, S. 60 f.; dies., Jugendverband, S. 240.

116 Bericht zur Blocksitzung vom 30. 11. 1949, abgedruckt bei Suckut, Blockpolitik, S. 560 f.

117 ACDP VI-052-0143, Protokoll PV, 27./28. 2. 1950, Beitrag Köhler, Sachsen (2. Verhandlungstag, S. 139f.).

118 So wandte er sich gegen die Fixierung auf einen Acht-Stunden-Tag, der Fütterung und Pflegezeiten einbezog, sowie die Beschränkung auf 300 Überstunden im Jahr. SAPMO VdgB 144, Protokoll Landesausschußsitzung Sachsen-Anhalt, 18. 7. 1950.

119 SAPMO VdgB 144, Neufassung Protokoll-Texte über die Fraktionssitzung vom 22. 12. 1949; siehe auch Protokoll Sekretariatssitzung der ZVdgB, 27. 7. 1950, ebd. Instruktiv zu Körtings Auftreten in der interfraktionellen Sitzung auch SAPMO DY 30 IV $2 / 2022 / 18$, Bl. 114-119, Aktenvermerk für Merker, 9. 12. 1949, hier Bl. 114-117. Demnach löste dieses Verhalten das weitere Vorgehen gegen Körting aus. 
zu sein, als er äußerte, daß er keine „Großbauern“ kenne, nur „reaktionäre“ und „fortschrittliche“ Bauern. Waren diese Angriffe von Körtings Anhängern noch leicht als vordergründig politisch motiviert zu erkennen, so versuchte man darüber hinaus, ihn als Amtsträger vor den bäuerlichen Mitgliedern zu diskreditieren ${ }^{120}$. Körting war im Grunde ein Opfer der allgemeinen "Säuberungspolitik“ nach dem III. Parteitag der SED, und man bemühte sich nicht umständlich darum, dies zu verheimlichen ${ }^{121}$; eher schon den Umstand, daß Vieweg ihn als Gegner in der VdgB kaltstellen wollte ${ }^{122}$. Körting, der im Sinne einer auf die Belange der Bauern bezogenen Agrarpolitik argumentierte, lief Gefahr, als Gegner des veränderten agrarpolitischen Gesamtkurses und der verschärften Bündnispolitik der SED gegenüber den sogenannten „Großbauern" eingestuft zu werden.

Auch die Führungsspitze der DBD war hinsichtlich des Landarbeiterschutzgesetzes gespalten. Nach außen hin stand ihre Parteipresse von Beginn an hinter dem Landarbeiterschutzgesetz und begrüßte es prinzipiell nach der Verabschiedung ${ }^{123}$. Paul Scholz formulierte noch einen Monat vor den Beratungen in der DWK im Juli 1949 gegenüber der SED-Landwirtschaftsabteilung Vorbehalte, die Körtings Kritik in nichts nachstanden. Im Gegensatz zu diesem erwuchs ihm daraus keinerlei Schaden. Das rührte sicherlich auch daher, daß Körtings Vorgehensweise es der CDU erlaubte, sich als „liebenswürdige und konziliante“ Verhandlungspartnerin zu profilieren. Dies zugelassen zu haben, provozierte ein rigoroses Vorgehen gegen Körting ${ }^{124}$. Die Verläßlichkeit von Scholz, dem Verbindungsmann zur SED in dieser Zeit, war hingegen über jeden Zweifel erhaben.

Ernst Goldenbaum stand nach außen ebenso wie in der Partei ganz hinter dem Gesetzesvorhaben. Seine Konformität mit der SED-Konzeption ist zweifellos durch den Umstand begünstigt worden, daß er sich zu dieser Zeit als Minister für das Landwirtschaftsressort gegen Herbert Hoffmann durchsetzen mußte, den die

120 So der Vorwurf, in illegale private Kreditvergaben verstrickt zu sein. Daher munitionierte Körting seine Verteidigungsrede mit vielerlei Anspielungen auf das illegale Finanzgebaren und Mittelverschwendung besonders des mecklenburgischen VdgB-Landesverbandes. SAPMO VdgB 144, Protokoll Landesausschußsitzung Sachsen-Anhalt, 18. 7. 1950.

$121 \mathrm{Vgl}$. Ebd.

122 Siehe dazu SAPMO VdgB 144, Rededisposition Viewegs für die außerordentliche 4. Hauptausschußsitzung, undatiert; ebd., Protokoll der 4. Hauptausschußsitzung. Körting wurde aus der Führung entfernt und genötigt, sein Volkskammermandat niederzulegen, schließlich nahm man ihn für ein Jahr in Untersuchungshaft. Es gereichte ihm - so wie zuvor Hoernle - zum Nachteil, daß er für einen eigenständigeren Kurs der VdgB gegenüber der SED eingetreten war. Zur Rolle Viewegs auch Scholz, Bauernopfer, S. 74, $112 \mathrm{f}$.; zu Jadasch und Hansch, die Vieweg unterstützten, SAPMO VdgB 144. Vgl. Wernet-Tietz, Bauernverband, S. 66 f., S. 223; Buss, Struktur, S. 113 f., wonach Körting auch in Widerspruch zur Gleichschaltung der ldw. Genossenschaften stand; Weber, Umgestaltung, S. 2854.

123 „Bauern-Echo“, 7. 5. 1949, S. 5. Auffälligerweise schwieg die Berichterstattung im „Bauern-Echo" bis zur endgültigen Beschlußfassung in der Volkskammer. Zweifellos hielt man das Thema für ungeeignet, das Mitgliederwachstum zu fördern. „Bauern-Echo“, 8. 12. 1948 mit Hinweis, daß die DBD erfolgreich Abänderungsvorschläge im Sinne der "werktätigen Bauern “ einbringen habe können. Wernet-Tietz, Bauernverband, S. 158.

124 SAPMO DY 30 IV 2/2022/18, Bl. 114-119, Aktenvermerk für Merker, 9. 12. 1949, hier Bl. 115. 
SED-Spitze gern statt seiner in der Regierungsmannschaft gesehen hätte. In der Wahrnehmung der Funktionäre und Mitglieder der DBD läutete diese Differenz zwischen Scholz und Goldenbaum eine jahrelange Rivalität ein ${ }^{125}$. Zu berücksichtigen ist jedoch die spezifische Gesprächssituation zwischen Scholz und den SEDVerantwortlichen. Die internen Beratungen in einem frühen Stadium erleichterten ihm, durch Kritik auf die endgültige Gesetzesfassung einzuwirken. Goldenbaum, für dessen Beteiligung an ähnlichen Aussprachen keine Hinweise vorliegen, hatte bei seinen Auftritten, etwa in der Blocksitzung am 30. November 1949, in der CDU und LDP das Gesetz attackierten, gar keine andere Wahl, als das Gesetz zu verteidigen, wenn er seinen Ministerposten behalten wollte ${ }^{126}$. Die DBD befand sich in einem Zwiespalt. Das Gesetz schädigte zweifellos die eigene Klientel, aus politischen Gründen gab es jedoch keine Alternative zur Zustimmung. Dies gilt um so mehr, wenn das Gesetz von sowjetischen Stellen oktroyiert wurde, da die DBD angesichts der Geringschätzung durch die SED auf die sowjetische Unterstützung existentiell angewiesen war.

Scholz' Argumentation suchte nach Wegen, wie die „werktätigen Bauern“ im Unterschied zu den „Großbauern“ von den Auswirkungen der Verordnung verschont werden könnten. Auf sie treffe der Begriff „landwirtschaftliche Unternehmer" nicht zu, wie ihn der Verordnungsentwurf zu dieser Zeit vorsah" ${ }^{127}$. Er befürchtete 1949, daß sich ein „grosser Teil der werktätigen Bauern wieder mit den Grossbauern [...] liiert und die bisher erreichten Erfolge hinfällig werden"128, wenn die Verordnung in dieser Form erlassen würde. Offensichtlich plante man zu dieser Zeit, die Verordnung so schnell wie möglich, unter Umständen noch vor der Ernte 1949 zu verkünden. Die langwierigen Beratungen zwischen allen Parteien und Organisationen in der DWK, die letztlich die Ablehnung der Gesetzesnovelle durch die Bauernschaft widerspiegelten, verhinderten dies. Auch Scholz empfahl eine Vertagung bis Herbst. Welchen offiziellen Standpunkt die DBD gegenüber der Verordnung einnehmen sollte, war Anfang Juni 1949 noch offen. Es zeichnete sich jedoch ab, daß die DBD sowohl die Haltung ihrer Mitglieder als auch die Positionen von CDU und LDP in ihr Kalkül einbeziehen mußte. Scholz gab zu bedenken, daß die bürgerlichen Parteien das Gesetz ablehnten und sie damit auf große Zustimmung unter der Bauernschaft stießen. Wenn das Gesetz noch vor dem für Juni 1949 geplanten I. Parteitag der DBD beschlossen würde, der unmittelbar nach dem Bauerntag stattfinde, welcher wiederum ein Forum für die Kritik der bürgerlichen Parteien biete, dann müsse die DBD das Gesetz wahrscheinlich ablehnen. Schließlich argumentierte er, daß die Landarbeiterschaft von

125 ACDP VI, unverz. Handakten Rose, Protokoll Sekretariat, 29. 11. 1950, Wortbeitrag Albrecht. So protegierte Goldenbaum in dieser Zeit, wenngleich erfolglos, Besler statt Scholz als Vorsitzenden der Wirtschaftskommission beim PV. ACDP VI-052-0143, Protokoll PV-Sitzung, 8. 11. 1949.

126 Vgl. Suckut, Blockpolitik, Tabelle 1.

127 Siehe SAPMO DY 30 IV 2/7/110, Bl. 130-136.

128 Ebd., Bl. 128 f., Mitteilung Abt. LW der SED, Krebaum, an Merker über eine Unterredung mit Scholz, Gen. Beuster (IG Land und Forst) und Genossin Wohlgemuth (VdgB) vom 1. 6. 1949, 2. 6. 1949. Danach auch das folgende. 
sich aus kein solches Gesetz fordern würde ${ }^{129}$. Dies zeigt, wie sehr sich die Landarbeiter dem dörflichen Milieu zugehörig fühlten und keineswegs bereit waren, einer Klassenzuschreibung zu folgen ${ }^{130}$. Andererseits unterstreicht es die Schwäche der IG Land und Forst als Interessenvertretung 131.

Gemeinsam mit der Vertreterin der VdgB, Wohlgemuth, forderte Scholz, die Maßgabe eines achtstündigen Arbeitstages zu prüfen, was im Grunde das Herzstück des Entwurfes traf. Insbesondere die Fütterungs- und Pflegezeiten könnten nicht ausschließlich im Rahmen dieser Arbeitszeitvorgabe verrechnet werden. Ebenso seien "werktätige Bauern“ außerstande, ihren verheirateten Landarbeitern eine möblierte Wohnung zu überlassen. Hier müsse man zwischen den Landarbeitern bei „Großbauern" unterscheiden, die häufig auf dem Hof wohnen würden, und denen bei „werktätigen“ Bauern, die bislang im Dorf ihr Quartier hatten.

Letztlich bewirkten all diese Einwände keine wesentliche Änderung, nur eine Verschiebung des Gesetzes. Lediglich einige sprachliche Schärfen, die gegen bäuerliche Privateigentümer gerichtet waren und Bauern pauschal als „Unternehmer“ bezeichneten, fehlten in der erlassenen Fassung 132. Das Gesetz wurde nach der Staatsgründung verabschiedet. Im Endeffekt war diese Verzögerung, durch die es erst im Wirtschaftsjahr 1950 zum Tragen kam, für SED wie DBD günstiger. Für die DBD verlängerte sich der Zeitraum ohne die negativen Folgen des Landarbeiterschutzgesetzes, in dem sie sich organisatorisch stabilisieren und die Mitgliederbasis ausdehnen konnte.

Neben der Gesetzesinitiative deutet auch der Gang der Verhandlungen auf massive sowjetische Einflußnahme hin. Außer dem innersten SED-Zirkel, der die sowjetischen Vorgaben zur Agrarpolitik umzusetzen hatte, gab es keinen Akteur, der vorbehaltlos hinter dem Entwurf stand. Sogar die IG Land und Forst, die durch das Gesetz aufgewertet wurde, mußte sich diskreditiert fühlen, weil die Gesetzentwürfe aufzeigten, wie sehr es ihr bislang mißlungen war, die bestehenden Tarifverträge durchzusetzen. Paul Merker warnte das Kleine Sekretariat erfolgreich davor, gleichzeitig mit dem Landarbeiterschutzgesetz eine Zusatzverordnung zum Schutz jugendlicher Landarbeiter zu erlassen, wie die SED-Jugendabteilung verlangt hatte. Dies könne die Verabschiedung des Landarbeiterschutzgesetzes weiter verschleppen. Er brachte vor, „daß eine besondere Zusatzverordnung den schon jetzt vorhandenen starken Widerstand unter den Bauern gegen das Landarbeiterschutzgesetz noch mehr verstärken würde“133.

129 Dies steht gegen die Behauptung von Dillwitz, Entwicklung, S. 61.

130 BA DK-1/8114, Bl. 149 ff., Bericht des MLF Sachsen an HVLF, 4. 2. 1949, hier Bl. 162; Bauerkämper, Neubauern, S. 120, 127; ders., Strukturumbruch, S. 78 ff.; Andeutungen auch bei Plaul, Veränderungen, S. $157 \mathrm{ff}$.

131 Deren Vorsitzender, Willi Beuster, bat darum, jene Passage aus der Präambel zu streichen, die ihr interessenpolitisches Versagen gegenüber den "landwirtschaftlichen Unternehmern" explizit hervorhob.

132 So wurde das Adjektiv "privat“ ebenso gestrichen wie die Bezeichnung der "landwirtschaftlichen Unternehmer" durch "Inhaber" oder "Leiter" ersetzt wurde. BA DC-20 I/ 3-1, Sitzung des Ministerrates, 24. 11. 1949, Bl. $91 \mathrm{ff}$., Gesetzentwurf in Anlage, Bl. $98 \mathrm{ff}$.

133 SAPMO DY 30 IV 2/7/110, Bl. 224, Vorlage Merker an Kleines Sekretariat, 2. 11. 1949. 
Die DBD reagierte verhalten auf das Gesetz. In den Landessekretariaten fanden zwar Debatten statt, doch hütete man sich davor, Gegenargumente wörtlich ins Protokoll zu nehmen ${ }^{134}$. Den Landessekretariaten wurde eingeschärft, daß bei den Funktionären politische „Klarheit“ herrschen müsse. Im mecklenburgischen Sekretariat betonte der Landesvorsitzende Ernst-Walter Beer, daß „unser Regierungsprogramm auch unser Programm ist und sein muß", und die DBD das Landarbeiterschutzgesetz voll anerkenne ${ }^{135}$. Offensichtlich gab es genügend Anlaß für diese unmißverständlichen Worte. In den Ortsgruppen und Kreisverbänden der DBD stieß das Gesetz ebenso auf Ablehnung, wie dies für die Bauernschaft insgesamt zu beobachten war. Es liegen keine Zeugnisse darüber vor, wie sich die knapp vier Prozent Land- und Forstarbeiter, die es bei Inkrafttreten in der DBD gab, verhielten. Ihre Interessen sind jedoch zweifellos von der gut 20 Prozent starken Mitgliedergruppe der Mittel- und Großbauern übertönt worden; zumal die zahlenmäßig schmal vertretenen „Großbauern" häufig eine innerdörfliche und innerparteiliche Führungsrolle behaupteten ${ }^{136}$. Nicht ohne Grund dürfte sich Paul Scholz veranlaßt gesehen haben, zunächst gegenüber der SED mit einer Ablehnung des Gesetzentwurfes aufgrund der Einstellung der Basis zu drohen.

Eine Episode illustriert den Unmut von Teilen der sächsischen Bauernschaft: Im Frühjahr 1950 waren im Landkreis Flöha 200 Bauern einem Versammlungsaufruf gegen das Landarbeiterschutzgesetz gefolgt, der angeblich von der VdgB stammte, die dann aber keinen Redner schickte. Eine Resolution, die zum Abdruck an die Zeitung "Der Freie Bauer" verschickt worden war, wurde nicht publiziert. Bereits Ende 1949 hatte sich ein anonymer Briefschreiber aus dem Landkreis an die VdgB wie DBD gewandt und so vehement gegen das Landarbeiterschutzgesetz gewettert, daß die zentrale SED-Landwirtschaftsabteilung zu ermitteln begann ${ }^{137}$. Auch die DBD wurde von ihm demnach als Anwalt gegen das Gesetz begriffen, obwohl im Kreis Flöha die CDU unter den Bauern wie in der VdgB dominierte ${ }^{138}$.

Offenbar schreckte die SED vor einer rückhaltlosen Durchsetzung des Gesetzeswerkes zurück, wahrscheinlich war sie dazu gar nicht in der Lage. Die SEDKritik richtete sich vor allem gegen den unzulänglichen Einsatz der IG Land und Forst. Die Arbeitgeber erschwerten Kontrollen, indem sie die Lohnbücher nicht ordnungsgemäß führten. Festgestellt wurde außerdem, daß die Beschäftigten verängstigt waren und es nicht wagten, „bei Überprüfungen den Kontrollorganen Auskunft über Art und Höhe ihrer Entlohnung zu erteilen bzw. bewußt fälschlicherweise behaupten, daß ihnen alle Arbeiten ordnungsgemäß bezahlt werden"139.

134 ACDP VI, unverz. Best. Sekretariatssitzungen der LV, Protokoll Sekretariat Sachsen-Anhalt, 9. 12. 1949.

135 ACDP VI, unverz. Best. LV Mecklenburg, Protokoll Sekretariat, 22. 11. 1949.

$136 \mathrm{Vgl}$. Tabelle 28.

137 SAPMO DY 30 IV 2/7/74, Bl. 111, Protokoll Abteilungsbesprechung, 13. 12. 1949.

138 ACDP VI-052-0143, Protokoll PV, 27./28. 2. 1950, S. 104-107. Noch 1951 war es der DBD nicht gelungen, daran etwas zu ändern, ACDP VI-052-222/1, Instrukteurbericht über KV Flöha, 8./9. 8. 1951.

139 Iwand, Wie wurde das Landarbeiterschutzgesetz bisher verwirklicht?, in: Arbeit und Sozialfürsorge, S. 232-233, Zitat S. 233. Siehe auch Frerich/Frey, Sozialpolitik, S. 116. 
Zweifellos begünstigte das Gesetz durch die Verteuerung der Arbeitskraft die Halbierung der Zahl der unselbständig Beschäftigten in der Landwirtschaft von 993878 im Jahr 1946 auf rund 435000 im Jahr 1953140. Probleme bei der Realisierung waren an der Tagesordnung. Anfangs wurden die Regelungen kaum umgesetzt, wie Überprüfungskommissionen der IG Land und Forst und des FDGB berichteten ${ }^{141}$. Noch 1952 forderte die Gewerkschaft ihre Mitglieder zur besseren Durchsetzung des Gesetzes auf und versuchte es propagandistisch zu verkaufen ${ }^{142}$. Altbauern im Kreis Greifswald wehrten sich schon im November 1949 gegen Hofbegehungen - sie führten damit die schon während des nationalsozialistischen Regimes eingeübten Verhaltensweisen fort, um den Kontrollanspruch der jeweiligen Herrschaftsträger zu begrenzen. Die dortigen Landarbeiter wußten entweder nicht von ihren Rechten oder wollten diese nicht wahrnehmen aus Furcht, ihre Arbeitsstelle zu verlieren ${ }^{143}$.

Einen mangelhaften Bekanntheitsgrad des Gesetzes registrierte auch die SKK Anfang 1950144. Semjonow monierte, daß die SED es nicht zur „Heranziehung der Landarbeiter auf ihre Seite " 145 ausnutze. Insgesamt kritisierte die SKK die schlechte Arbeit der SED unter der Bauernschaft, selbst arme Bauern würden gegen die MAS auftreten ${ }^{146}$. Ein positiver Effekt des Gesetzes auf die Mitgliederentwicklung der SED blieb 1950 aus. Sowohl die Mitglieder- und Kandidatenzahl der Landarbeiter war rückläufig - bis Mai 1950 hatte sie sich um 6000 auf 56400 verringert - als auch die der Bauern. Sie verminderte sich im Laufe eines Jahres um rund 7000 auf $97100^{147}$. Noch 1957 wurde das Landarbeiterschutzgesetz auf größeren Höfen nicht überall angewandt. Opponenten der SED auf dem Land machten es weiterhin zum Gegenstand ihrer Vorwürfe ${ }^{148}$.

140 BA DK-1/8152, Bl. 10, Stand vom 29. 1. 1946; BA DQ-2/2122, Vermerk vom 1. 12. 1953; SAPMO DY 30 IV $2 / 7 / 243$, Bericht über die Parteiarbeit auf dem Lande, undatiert [1950]: 1013000 Land- und Forstarbeiter, 950000 Bauern.

141 Kontrollen der IG Land und Forst zufolge hatten im Jahr 1950 zwar 75\% der überprüften Betriebe Verträge abgeschlossen, doch nur 20-30\% hielten diese ein. Schulz, Probleme, S. 20, der allerdings den bis Mitte der fünfziger Jahre anhaltenden Widerstand unzulässig nur auf die Großbauernschaft verengt. Bauerkämper, Aufwertung, S. 256.

142 Siehe Landarbeiterschutzgesetz und das Leben des Landarbeiters von gestern und heute, bes. S. $13 \mathrm{f}$.

143 SAPMO DY 30 IV 2/7/113, Bl. 40 f., Schreiben Abt. LW an Abt. Arbeit und Sozialfürsorge, 25. 11. 1949. Zum Osthavelland ebenso Bauerkämper, Aufwertung, S. 255.

144 Badstübner/Loth (Hg.), Wilhelm Pieck-Aufzeichnungen, S. 326-328, Besprechung 23. 1. 1950 bei Tschuikow, hier S. 326.

145 Ebd., S. 328-331, hier S. 330, Bericht Semjonows, 24. 1. 1950.

146 Ebd., S. 326, Besprechung am 23. 1. 1950 bei Tschuikow.

147 SAPMO DY 30 IV 2/7/243, Bericht über die Parteiarbeit auf dem Lande, undatiert [1950], Bl. 26-58, hier Bl. $26 \mathrm{f}$.

148 ACDP VI-052-1355, Protokoll Sekretariat BV Schwerin der DBD, 13. 5. 1957. 


\section{Interessenpolitik der DBD?}

In seiner Studie über die Frühzeit der DBD kommt Wernet-Tietz zu dem Ergebnis, daß sich die DBD im Rahmen der „produktionsfördernden, leistungshebenden und den Plan konkretisierenden Forderungen und Initiativen [...] für wirtschaftliche, soziale und kulturelle Belange der Bauern " ${ }^{149}$ als Interessenvertretung einsetzte. Ihren Bemühungen um einen Auf- und Ausbau des landwirtschaftlichen Schulwesens und zur finanziellen Förderung und Gleichstellung des landwirtschaftlichen Nachwuchses mit dem der Industrie war mehr Erfolg beschieden als manchen agrarwirtschaftlichen Vorstößen. Eine Vorlage der DBD zur Verbesserung des landwirtschaftlichen Schulwesens für den Unterausschuß des Deutschen Volksrates wurde am 26. April 1949 mit unerheblichen Änderungen als Grundlage des weiteren Vorgehens akzeptiert. Eine Verordnung über die Vergabe von Stipendien, die am 1. Januar 1950 in Kraft trat, eröffnete auch Kindern von Klein- und Mittelbauern die Chance zum agrarwissenschaftlichen Studium ${ }^{150}$. Beide Beispiele stießen sicher auf den Konsens aller Parteien. Denn sie versprachen Personallücken an landwirtschaftlichen Fachkräften zu schließen, die aus einer nach politisch-ideologischen Kriterien geleiteten „Säuberungs“-Politik gegen oft als „bürgerlich“ diffamierte Spitzenkräfte resultierten.

Darüber hinaus ist festzustellen, daß die DBD bei ihren agrarpolitischen Vorstößen nicht nur die Klein- und Neubauern im Blick hatte, sondern auch als Fürsprecher der potenteren Gruppe der Mittelbauern von 15 bis 20 ha auftrat, die gerade 1950 Gefahr lief, schon zu den „Großbauern“ gezählt zu werden. Das „Gesetz über die Verbesserung der Versorgung der Bevölkerung und über die Pflichtablieferung landwirtschaftlicher Erzeugnisse 1950"151 belastete laut internen Stellungnahmen der DBD die mittleren Betriebe zu stark. Sie bedeutete für 10-20 ha große Betriebe „eine wesentliche Erhöhung des Ablieferungssolls"152, wie die DBD gegenüber dem SKK-Mitarbeiter Buschmanow im Juni 1950 vorbrachte. Aufgrund der Differenzierungspraxis zwischen den Ländern und Differenzierungsfehlern in Kreisen und Gemeinden erhöhe es für viele Betriebe das bisherige Soll um 40, 50 oder sogar mehr als 60 Prozent. „Die Bauern der Betriebsgrössengruppe von 10-20 ha und natürlicherweise auch die über 20 ha sind weitgehend der Auffassung, dass diese starke Erhöhung eine politische Massnahme gegen sie ist, die durchgeführt wurde, um sie zu ruinieren." Im Bewußtsein, daß unpopuläre agrarwirtschaftliche Maßnahmen die Stimmung gerade auch gegen die Partei, die den Landwirtschaftsminister stellte, trüben würden, hielt man ,die Sollerhöhung für viele Betriebe in der Grössenklasse von 10-20 ha in der Tat für schwer tragbar". Daher setzte sich die DBD bei der SKK und beim Politbüro für Erleichterungen in der Größenklasse von 10-20 ha ein. Als Reaktion dar-

149 Wernet-Tietz, Bauernverband, S. 156.

150 Siche „Bauern-Echo“, 28. 4. 1949 und 1.1. 1950; Wernet-Tietz, Bauernverband, S. 156.

151 Erlassen am 22. 2. 1950; GBl. DDR 1950, 15. 3. 1950, S. $163 \mathrm{ff}$.

152 ACDP VI-052-0001c, Stellungnahme zu einigen politischen Problemen lt. Schreiben vom 30. 5. 1950. Wernet-Tietz stuft das Gesetz schon in dieser Form als Erfolg der DBD für die kleineren und mittleren Bauern ein, Wernet-Tietz, Bauernverband, S. 157. 
auf, die einen Erfolg für die DBD bedeutete, kann die Mitte August erlassene „Verordnung über die Ermäßigung des Ablieferungssolls von Getreide für die Bauernwirtschaften in der Größe von 10-15 ha" gelten ${ }^{153}$. Sie brach die feste Unterteilung der Größengruppe von 10-20 ha auf ${ }^{154}$. Die DBD vertrat auch später, im September 1950 gegenüber Tschuikow den Wunsch, zusätzliche Zwischenstufen in den bisherigen Betriebsgrößengruppen einzuführen, um das Ablieferungssoll "noch besser den Leistungsmöglichkeiten der einzelnen Bauernwirtschaften anzupassen"155. Die DBD argumentierte in Kenntnis der Notlage vehementer als die SED mit betriebswirtschaftlichen Gründen der Bauern.

Völligen Schiffbruch erlitt die DBD hingegen mit dem Anliegen, sowjetische Unterstützung für die Durchführung des Bodenreformbauprogrammes nach dem SMAD-Befehl $209 \mathrm{im}$ Jahr 1950 zu erhalten ${ }^{156}$. Ihr Bemühen um eine Weiterverfolgung oder Neuauflage dieses einst mit enormem Propagandaaufwand vertretenen Programms für die eigene Klientel der Neubauern war vergebens. Offensichtlich betrachteten SKK und SED die bis dahin getroffenen Regelungen zur Entschuldung von Klein-, Neu- und Mittelbauern als Abschluß des Bodenreformbauprogramms. So sah das noch vor den Wahlen 1950 am 8. September erlassene "Gesetz über Entschuldung und Kredithilfe für Klein- und Mittelbauern“ vor, den Neubauern, die bis Ende Juni 1950 Bodenreformkredite aufgenommen hatten, die Hälfte der Zahlungsverpflichtungen zu erlassen, sofern diese nicht schon im Zuge der Währungsreform 1948 umbewertet worden waren. Denn auf Anregung der DBD hatte die DWK Ende 1948 beschlossen, bei Kreditschulden, die aus der Bodenreform herrührten, eine Abwertung auf ein Fünftel vorzunehmen. Eine weitere Minderung räumte die „Anordnung zur Erleichterung der Entschuldung der Bauernwirtschaften“ vom 5. Oktober 1949 ein. Das Gesetz vom September 1950 galt auch für jene bäuerlichen Gruppen, die Kredite zur Behebung von Kriegsschäden an Wirtschafts- und Wohngebäuden aufgenommen hatten, die also nicht unmittelbar in Zusammenhang mit der Bodenreform standen. Außerdem entschuldete es Altsiedler, die vor dem 8. Mai 1945 Grundstücke oder Siedlerstellen gekauft hatten ${ }^{157}$. In zeitlicher Nähe zu den Wahlen 1950 wurde eine Reihe von Anliegen entschieden, die bereits in den ersten Programmentwürfen und im Programm der DBD von 1949 auftauchten. Diesen Umstand hob die DBD im erneuten Bittschreiben an die SKK dankend hervor ${ }^{158}$. Dennoch blieben die Wahlgeschenke der SKK bescheiden und der Erfolg der Bauernpartei begrenzt.

Wie bereits im Parteiprogramm von 1949 gefordert, bekräftigte die DBD gegenüber der SKK, für wie notwendig sie die rasche Einführung einer landwirtschaftlichen Einheitssteuer hielt, die auf dem Hektar-Ertragswert und einer ein-

153 GBI. DDR 1950, 23. 8. 1950, S. 845.

154 Der Einsatz für diese Erleichterung kann daher nicht nur als Beweis für Kurt Viewegs "Interessenpolitik“ gewertet werden. So Scholz, Bauernopfer, S. 119.

155 ACDP VI-052-0001c, PV der DBD an Tschuikow, 26. 9. 1950.

156 Ebd.

157 Zur Frage der Entschuldungen: Wernet-Tietz, Bauernverband, S. 158; Schulz, Probleme, S. 16.

158 ACDP VI-052-0001c, PV der DBD an Tschuikow, 26. 9. 1950. 
heitlichen Bonitierung basieren sollte. Sie strebte danach, von der bisherigen progressiven Besteuerungspraxis nach Hektargröße ohne Feststellung eines neuen Einheitswertes wegzukommen, da diese zu Lasten der mittleren und größeren Betriebe ging ${ }^{159}$. Ebenso setzte sich die DBD gegenüber der SKK für eine Neuregelung der Sozialversicherungsbeiträge der Bauern ein, die sich an Größe und wirtschaftlicher Leistungsfähigkeit der Betriebe bemessen sollten ${ }^{160}$. Offenbar war der SKK an einer zügigen Regelung dieser Fragen jedoch nicht gelegen. Fritz Weißhaupt monierte im November 1951 in der Volkskammer erneut, daß das Einheitssteuergesetz immer noch in den Kinderschuhen stecke ${ }^{161}$. Vielleicht hielt man neue steuerrechtliche Regelungen auch bewußt als Druckmittel noch zurück und behielt sich solche für jene Phase vor, die eine Kollektivierung offen propagierte ${ }^{162}$. Bereits die Steuergesetzentwürfe von 1950 enthielten jedenfalls eine Begünstigung des volkseigenen und dorfgenossenschaftlichen Sektors, 1952 sollte das Gesetz vollends zur Bildung von LPG instrumentalisiert werden ${ }^{163}$.

Ebenso verzögerte sich die Durchführung der am 22. November 1951 vom Ministerrat beschlossenen Verringerung der Sozialversicherungsbeiträge für Bauern. Zwar verlangte die DBD auf der Volkskammersitzung am 19. März 1952 vom LDP-geführten Finanzministerium eine beschleunigte Umsetzung, erhielt jedoch im Juni 1952 eine abschlägige Antwort des Ministers Hans Loch. Er schloß eine Senkung 1952 aus. Erst in Verbindung mit der Genossenschaftsbildung war eine Ermäßigung dann doch finanzierbar. Insgesamt scheiterten die Anstrengungen der DBD um die einzelbäuerlichen Interessen in diesen beiden wesentlichen Punkten, Besteuerung und Sozialversicherung. Im Gegenteil, als zentrale Regelungsbereiche moderner Staatlichkeit wurden sie später für die sozialistische Umwälzung benutzt.

Die DBD vertrat eine Reihe von interessenpolitischen Zielen, insbesondere diejenigen für die Gruppe der Bauern mit 15 bis 20 ha, im Grunde nur intern, wenngleich auch gegenüber der SKK. Sie hütete sich davor, diese Anliegen in der Parteipresse zu verbreiten, und schloß dadurch jeden Verdacht aus, für eine bäuerliche Interessenpolitik ihre Integrität als loyale Gehilfin der SED riskieren zu wollen. Es ist nicht mehr im einzelnen festzustellen, ob die gesetzgeberischen Erfolge tatsächlich auf ihren Einsatz zurückzuführen sind oder auch ohne sie beschlossen

159 Die am 1. 12. 1948 von der DWK beschlossene Steuerreform legte eine nach Betriebsgrößen differenzierte Steuer fest. Großbauern zahlten je Hektar 30\% mehr Steuern als Kleinbauern. Scholz, Bauernopfer, S. 78; Schulz, Entwicklung, S. 214; präziser Kahle, Steuerpolitik, S. 101-108, bes. zur Landwirtschaft, S. 114-119, wobei Kahle aktengestützt sowjetischen Einfluß auf die Regelung vom 1. 12.1948 hervorhebt. „Verordnung zur Änderung und Ergänzung von Steuergesetzen“ 1.12. 1948, abgedruckt ZVOBl. 1949, Nr. 29 , S. $235 \mathrm{ff}$.

160 ACDP VI-052-0001c, PV der DBD an Tschuikow, 26. 9. 1950; Wernet-Tietz, Bauernverband, S. 157.

161 Ebd., S. 157; zum Neuregelungsversuch 1949 und dem Scheitern 1950, Kahle, Steuerpolitik, S. 163-178.

162 Die Steuerpolitik war als Mittel der „Klassendifferenzierung“ auch in Viewegs „Maßnahmenkatalog" festgehalten: BA DC-15/737, Bl. 399-415, Bericht über Reise nach Polen, 6. 12. 1948, an Rau, Bl. $414 \mathrm{f}$.

163 Kahle, Steuerpolitik, S. $167 \mathrm{ff}$. 
worden wären. Hier ist zu bedenken, daß die Kontrolle von SMAD/SKK und SED in jedem Fall so weit reichte, daß die DBD nichts in die politische Diskussion auf oberster Ebene einbrachte, was außerhalb des Vorstellungsbereichs der Machthaber lag. Auch die ursprüngliche Ablehnung des Landarbeiterschutzgesetzes seitens der $\mathrm{DBD}$, welches sie dann aber mittrug, steht exemplarisch für dieses Verhalten. Da sowjetischen Stellen und SED aber an der Etablierung der DBD zur eigenen Herrschaftssicherung gelegen war, ist das Engagement der Bauernpartei nur instrumentalisiert worden. Dennoch wirkte sie als Korrektiv im interessenpolitischen Auftrag der Bauernschaft. Sie versuchte Profil dadurch zu gewinnen, daß sie Umsetzungsprobleme, die sich vor Ort ergaben, aufgriff, diese auch betriebswirtschaftlich unter die Lupe nahm und sowohl interessenbezogen wie herrschaftsstabilisierend agierte, indem sie Härten bei den Klein- und den größeren Mittelbauern ausräumen wollte. Insofern versuchte die DBD tatsächlich, dem Programm des I. Parteitages 1949 zu folgen, das sich auf die „werktätigen“ Kleinund Mittelbauern bezog und nur die „Großbauern“ explizit aussparte. Das dehnbare Konstrukt des „werktätigen Bauern“, das sich im Prinzip auch dazu eignete, Mittelbauern auszugrenzen, wurde in der DBD 1950 so gut wie nicht klassenkämpferisch mißbraucht. Zu diesem Zeitpunkt überwogen in der Partei meist noch wirtschaftsbezogene Kriterien. Sie war in erster Linie damit beschäftigt, die "Großbauern" aus den Funktionsrängen in Orten und Kreisen zu verdrängen. In dieser „Säuberungspolitik“ hinkte die DBD der SED-Praxis hinterher und arbeitete phasenverschoben zu dieser noch mit bäuerlichen Gruppen, die die sozialistische Partei offiziell schon ausgegrenzt hatte. Wie der Einsatz der DBD bei der SKK für die Mittelbauern von 15 bis 20 ha beweist, agierte die DBD damit jedoch innerhalb des Aufgabenspektrums, das ihr die SKK zuschrieb.

\section{Fazit}

Mit der Staatsgründung war in der Wahrnehmung großer Teile der bäuerlichen Bevölkerung eine weitere wirtschaftliche und finanzielle Belastung verknüpft. So ist für die Jahre von 1950 bis 1952 charakteristisch, daß prinzipiell alle bäuerlichen Größenklassen von jährlichen Sollerhöhungen betroffen waren; danach wurde dieser Hebel zur Produktionssteigerung nicht mehr eingesetzt. Während für Kleinbauern unter Fürsprache der DBD Sonderregelungen oder nachträgliche Erleichterungen geschaffen wurden, interpretierten die mittel- bis großbäuerlichen Betriebe die SED-Agrarpolitik als gezielt gegen sie gerichtet.

Die aus primär ernährungspolitischen Motiven ergriffenen Maßnahmen wie der Wechsel zur Hektarveranlagung und die Ausdehnung der Ackeranbaufläche erhöhten den wirtschaftlichen Druck in den Dörfern. Dieser bewirkte einerseits eine Solidarisierung unter den bäuerlichen Schichten im Ort, da alle - wenn auch nicht in gleicher Weise - betroffen waren. Die traditionalen Bindungen überwogen und definierten sich weitgehend durch die Zugehörigkeit zur gleichen Berufsgruppe, wobei der Bauernschaft die Schichtunterschiede untereinander durchaus bewußt waren - darin war sie jahrhundertelang eingeübt. Solange diese 
Interpretation der sozialen Wirklichkeit Bestand hatte, blieb der politisierende Einfluß der SED und auch der DBD, die die bäuerliche Bevölkerung durch „Differenzierung" aufzuspalten suchten, begrenzt. Waren die traditionalen Ausgleichsmechanismen im sozialen Verhältnis gestört, etwa dann, wenn die altbäuerliche Bevölkerung der neubäuerlichen jegliche Hilfe verwehrte, so hatte die auf Trennung und eine Klassendifferenzierung nach ökonomisch-politisierten Kriterien ausgerichtete Strategie der SED-Agrarpolitik durchaus Chancen, bei jenen Teilen der Dorfeinwohner auf Akzeptanz zu stoßen, die für die SED-Versprechungen empfänglich geworden waren. Widerstände gegen die verordnete Agrarpolitik und politische Auseinandersetzungen im Dorf wurden von der SED und jenen Teilen der DBD, die bereits auf SED-Kurs gebracht worden waren, in erster Linie ideologisch zu erklären versucht: Friktionen und Probleme waren auf den Einfluß des „Klassengegners“ zurückzuführen, sein Verhalten wurde als „Sabotage" kriminalisiert.

Das Jahr 1950 markiert in diesem Zusammenhang eine Zäsur. Die Anzeichen für eine latente Sozialisierung in der Agrarpolitik mehrten sich, als die SED den staatlichen Sektor nicht nur ideologisch aufwertete, sondern auch anteilsmäßig auszubauen suchte. Dies ist abzulesen am Flächenzuwachs der volkseigenen Betriebe, die verlassene, unbewirtschaftete Flächen vereinnahmten, aber auch die Produktionsflächenausdehnung, wie sie im Konzept der Erreichung von „Friedenshektarerträgen " vorgesehen war, realisieren sollten ${ }^{164}$. Gerade letzteres belegt die eklatante Fehleinschätzung der tatsächlichen betriebswirtschaftlichen Leistungsfähigkeit des volkseigenen Sektors bis dahin. Dabei liegt ein Mischungsverhältnis von intentional betriebener Sozialisierungspolitik einerseits und dem Auffangen des ökonomischen Problemdrucks - vor allem bei den desolaten Neubauernbetrieben - durch den volkseigenen Bereich andererseits vor.

Der Flächenanteil des sozialistischen Sektors wuchs aufgrund seiner Funktion als Auffangbecken für mittelbar selbst verursachte Schwierigkeiten. Das prototypische Beispiel für diese Entwicklung ist der Umgang mit dem Problem der nichtbewirtschafteten Flächen, die im Jahr 1950 beträchtliche Ausmaße annahmen. Denn viele Flächen wurden dem volkseigenen Sektor einverleibt, nicht weil die SED in dieser Phase eine aktive und offene Enteignungspolitik betrieb, sondern weil die Produktionsbedingungen so gestaltet waren, daß sie die Rentabilität von Privatbetrieben schmälerten und deren langfristiges Überleben gefährdeten, obgleich diese aus ernährungspolitischen Gründen noch eine tragende Säule der Agrarwirtschaft bilden sollten. Daher versuchte man Einzelbauern mit Sollermäßigungen und Vergünstigungen anzuregen, zusätzliche Flächen zu bewirtschaften. Die DBD übernahm es, diesen Weg zu popularisieren und die Bereitschaft dazu unter den Bauern zu erwirken. Allerdings wäre dies höchstwahrscheinlich nicht erfolgt, wenn die volkseigenen Betriebe in der Lage gewesen wären, diese Aufgabe ihrerseits zu erfüllen.

Parallel zur latenten Sozialisierungspolitik von 1950 wurden im gleichen Jahr eine Reihe von Maßnahmen in Angriff genommen, die die DBD an ein „höheres“

164 Scherstjanoi, „Friedenshektarerträge“, S. 336. 
politisches Niveau im Sinne des Marxismus-Leninismus heranführen und für künftige Aufgaben wappnen sollten. Hierzu zählte die „Säuberung“ der Funktionsposten von "Großbauern". Zwar war deren Aufnahme in die Partei nicht mehr vorgesehen, auch sollten die vorhandenen ausgegrenzt werden. Eine systematisch angelegte rigorose Verdrängung wurde jedoch weder angestrebt noch durchgeführt. Die DBD versuchte, das Engagement der „Großbauern“ weg vom Einfluß in der Partei auf ihre Mitarbeit in der "Nationalen Front“ umzulenken.

Einer gewissen Toleranz gegenüber den einfachen Mitgliedern stand auf der anderen Seite die Gleichschaltung der Funktionäre auf Landesebene gegenüber. 1948 hatte man erhebliche Differenzen in den Landesverbänden bzw. zwischen ihnen und der Zonenleitung zu Kernfragen wie den "Großbauern" und zur Haltung gegenüber den "Genossenschaften " 165 zwar als politische Fehler gebrandmarkt, jedoch keine Maßnahmen daraus abgeleitet. Wer dabei jedoch Rückgrat bewies, sogar in Parteivorstandssitzungen abweichende Meinungen äußerte und das Mißfallen der SED erregte, wurde bestraft. So erging es Richard Köhler, der 1948 im Auftrag der SED in die DBD übergetreten war, und einer Reihe weiterer Parteivorstandsmitglieder der Gründungszeit. Insbesondere jene, die sich wie Arthur Pech 1949 dafür eingesetzt hatten, daß die DBD gemäß Parteiprogramm als „selbständige politische Partei der werktätigen bäuerlichen Bevölkerung und der mit ihr verbundenen Berufsschichten " 166 auftrete, wurden in internen Diskussionen zwar mit umständlichen Worten zurechtgestutzt, aber nicht als politische Gegner stigmatisiert ${ }^{167}$. Im Unterschied dazu ging man nach der Aufbauphase der Partei 1950 gezielt zur Entfernung politisch nonkonformer Personen über. Auffälligerweise fiel ihre Sanktionierung in eine Zeit, die mit dem Machtverlust Goldenbaums ab Herbst 1950, der seine schützende Hand über Parteigründer hielt, und dem Aufstieg Berthold Roses als Generalsekretär zusammenfiel168.

Vor allem im Zusammenhang mit den Wahlvorbereitungen 1950 war die DBD im Innern ideologisch auf Linie gebracht worden. Die Pflicht zur Mitarbeit aller Funktionäre im Wahlkampf stand außer Frage, obwohl in Sachsen und SachsenAnhalt bisweilen die Auffassung vertreten wurde, daß der Erfolg der Parteiarbeit durch das Engagement in der „Nationalen Front“ gefährdet sei, und vor der Ein-

165 Damit bezeichnete man im Parteijargon alle ldw. Genossenschaften in Abgrenzung zur VdgB und inkriminierte damit eine zu positive Haltung ihnen gegenüber. ACDP VI-0520141, Protokoll PV, 3. 12. 1948.

166 Programm und Statut der DBD 1949, zitiert nach Broschüre in ACDP VI-052-0004.

167 ACDP VI-052-0143, Protokoll PV, 8. 11. 1949, Ausführung Scholz, S. $29 \mathrm{f}$.

168 Köhler meinte auf der PV-Sitzung am 27./28. 2. 1950, man dürfe „ruhig mal den Westsender" hören, ACDP VI-052-0143, Protokoll PV 27./28. 2. 1950. Beleg für Sanktionierung von Parteivorstandsmitgliedern in: ACDP VI-052-0144, Protokoll PV, 1. 2. 1951. Gegen eine Reihe von Parteiveteranen waren Parteischiedsgerichtsverfahren eingeleitet worden, darunter Paul Reder, mecklenburgisches Gründungsmitglied (Ausschluß, jedoch offensichtlich nicht durch politisch unkonformes Verhalten begründet), Richard Köhler (Aberkennung von Funktionen für drei Jahre), Einleitung eines Parteiverfahrens gegen Thiem. ACDP VI, unverz. Handakte Rose, Protokoll Sekretariat, 29. 11. 1950. 
führung der Einheitsliste gewarnt wurde169. Angriffe in der DBD gegen „fortschrittliche“ Funktionäre sollte man daher „bis zur letzten Endkonsequenz erledigen. Alle Versuche, fortschrittliche Funktionäre zu isolieren, müssen gestoppt werden ${ }^{“ 170}$. Im innersten Zirkel waren die marxistisch-leninistischen Grundübungen in Kritik und Selbstkritik nachweislich erstmals im November 1950 gegenüber Goldenbaum exerziert worden.

169 ACDP VI-052-0143, Protokoll PV, 27./28. 2. 1950, Beitrag Scholz, S. 26 f.

170 ACDP VI-052-0520, Protokoll Sekretariat, 19. 4. 1950. 